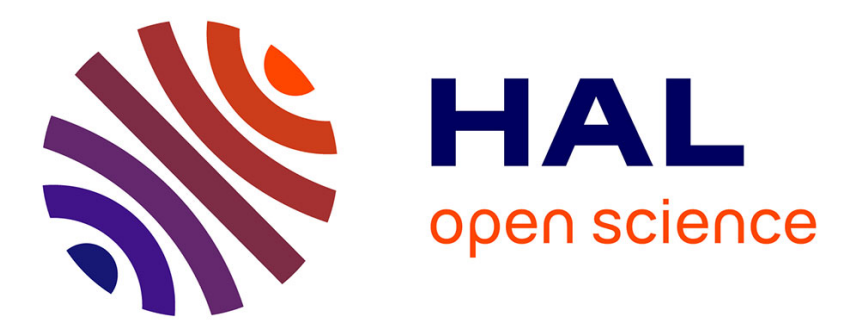

\title{
Deterministic solution of the kinetic theory model of colloidal suspensions of structureless particles
}

Guillaume Maîtrejean, Amine Ammar, Francisco Chinesta, Miroslav Grmela

\section{To cite this version:}

Guillaume Maîtrejean, Amine Ammar, Francisco Chinesta, Miroslav Grmela. Deterministic solution of the kinetic theory model of colloidal suspensions of structureless particles. Rheologica Acta, 2012, 51 (6), pp.527-543. 10.1007/s00397-011-0609-3 . hal-01061268

\section{HAL Id: hal-01061268 \\ https://hal.science/hal-01061268}

Submitted on 5 Sep 2014

HAL is a multi-disciplinary open access archive for the deposit and dissemination of scientific research documents, whether they are published or not. The documents may come from teaching and research institutions in France or abroad, or from public or private research centers.
L'archive ouverte pluridisciplinaire HAL, est destinée au dépôt et à la diffusion de documents scientifiques de niveau recherche, publiés ou non, émanant des établissements d'enseignement et de recherche français ou étrangers, des laboratoires publics ou privés. 


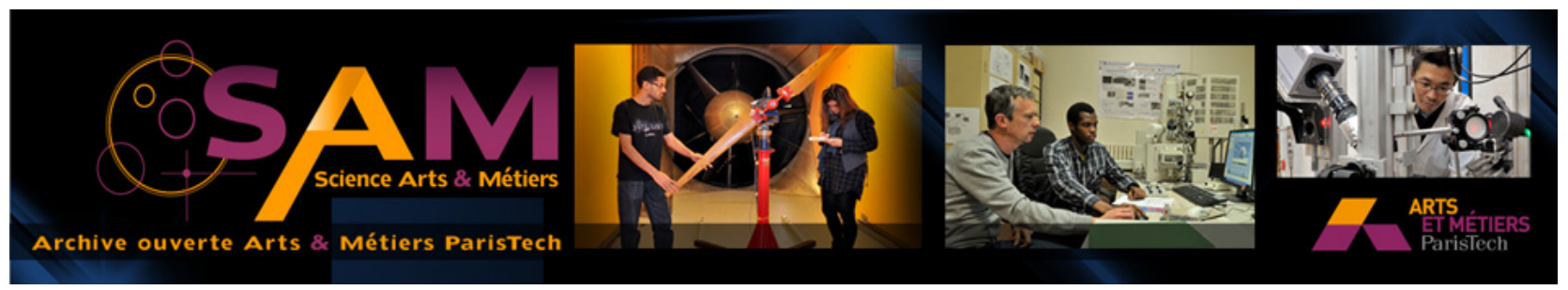

Science Arts \& Métiers (SAM)

is an open access repository that collects the work of Arts et Métiers ParisTech researchers and makes it freely available over the web where possible.

This is an author-deposited version published in: http://sam.ensam.eu

Handle ID: .http://hdl.handle.net/10985/8466

\section{To cite this version :}

Guillaume MAîTREJEAN, Amine AMMAR, Francisco CHINESTA, Miroslav GRMELA -

Deterministic solution of the kinetic theory model of colloidal suspensions of structureless particles - Rheologica Acta - Vol. 51, n6, p.527-543 - 2012 


\title{
Deterministic solution of the kinetic theory model of colloidal suspensions of structureless particles
}

\author{
Guillaume Maîtrejean • Amine Ammar · \\ Francisco Chinesta $\cdot$ Miroslav Grmela
}

\begin{abstract}
A direct modeling of colloidal suspensions consists of calculating trajectories of all suspended objects. Due to the large time computing and the large cost involved in such calculations, we consider in this paper another route. Colloidal suspensions are described on a mesoscopic level by a distribution function whose time evolution is governed by a Fokker-Plancklike equation. The difficulty encountered on this route is the high dimensionality of the space in which the distribution function is defined. A novel strategy is used to solve numerically the Fokker-Planck equation circumventing the curse of dimensionality issue. Rheological and morphological predictions of the model that includes both direct and hydrodynamic interactions are presented in different flows.
\end{abstract}

G. Maîtrejean ( $\square)$

Laboratoire de Rhéologie, UJF-INPG-CNRS, 1301 rue de la piscine, Domaine universitaire, BP 53, 38041 Grenoble cedex 9, France e-mail: guillaume.maitrejean@ujf-grenoble.fr

\author{
A. Ammar \\ Arts et Métiers ParisTech, 2 Boulevard du Ronceray, \\ BP 93525, 49035 Angers cedex 01, France \\ e-mail: amine.ammar@ensam.eu \\ F. Chinesta \\ EADS Corporate Fundation International Chair, \\ Ecole Centrale de Nantes, 1 rue de la Noe, \\ BP 92101, 44321 Nantes cedex 3, France \\ e-mail: francisco.chinesta@ec-nantes.fr \\ M. Grmela \\ École Polytechnique de Montréal, C.P. 6079 suc. \\ Centre-ville, Montréal, H3C 3A7, QC, Canada \\ e-mail: miroslav.grmela@polymtl.ca
}

Keywords Colloidal suspensions $\cdot$ Smoluchowski equation $\cdot$ Proper generalized decomposition

\section{Introduction}

Colloidal systems are encountered in many natural and industrial processes such as blood, foodstuffs, or paints where the presence of particles modifies in a non-linear way the behavior of the fluid. These suspended particles are subjected to different kind of forces coming from the fluid drag, inter-particles potentials, particlessolvent interaction, Brownian forces, etc. Thus, usual descriptions lie in discrete description of each particle and usual models proceed within the Brownian dynamics (BD) or Stokesian dynamics (SD) (Brady and Bossis 1988) framework. When the solvent is considered as continuum and the Reynolds number of particles is small, SD is able to account for interactions between colloidal suspension mediated by the solvent, the socalled HI. As soon as the position and eventually the momentum of all the colloidal particles are known, the virial stress could be computed without any major difficulty. However, the tracking of all the particles in the population implies significant computing time in order to limit the inherent statistical noise that stochastic simulations induce (Brady 2001).

An alternative to the $\mathrm{BD}$ and $\mathrm{SD}$ lies in deriving a continuous and deterministic kinetic theory description. This route leads to the so-called Fokker-Planck equation that characterizes the microstructure through the phase space distribution function $\Psi(\boldsymbol{x}, \boldsymbol{R}, \boldsymbol{V})$. On a more macroscopic level, the Smoluchowski description 
replaces the Fokker-Planck's formalism by a pair correlation function $g(\boldsymbol{x}, \boldsymbol{R})$ related to $\Psi(\boldsymbol{x}, \boldsymbol{R}, \boldsymbol{V})$ by

$g(\boldsymbol{x}, \boldsymbol{R})=\int \mathrm{d} \boldsymbol{V} \Psi(\boldsymbol{x}, \boldsymbol{R}, \boldsymbol{V})$

where $\boldsymbol{x}$ is the position vector, $\boldsymbol{R}$ the vector connecting two particles, and $\boldsymbol{V}$ their relative velocity. The difference between the Fokker-Planck and the Smoluchowski formulation lies in the elimination in $g(\boldsymbol{x}, \boldsymbol{R})$ of fast processes related to inertia (motion of the suspended particles through the fluid). By the simplifying assumptions made for the definition of the Smoluchowski equation, where only binary hydrodynamic interactions are considered, we may expect that the suspensions are rather dilute as the higher-order correlations are neglected. Furthermore, we would like to emphasize that colloidal systems have been widely studied (see, for example, reviews from Stickel and Powell (2005) and more recently Morris (2009)) from a theoretical (Batchelor 1977; Brady and Morris 1997; Lionberger and Russel 1997; Stickel et al. 2006; Wilson 2005) or experimental (Gao et al. 2010) point of view as well as numerically (Bergenholtz et al. 2002; Foss and Brady 2000; Phung et al. 1996). However, the major part of this work addressed concentrated system which cannot be accurately reproduced by Smoluchowski equation as mentioned previously. Nonetheless as we will see in this paper, our approach gives relevant results when comparing to theory.

The Smoluchowski formulation is defined on both physical space $\boldsymbol{x}$ and configuration space $\boldsymbol{R}$; thus, a strategy for solving high-dimensional problems is needed. For that reason, in the literature, the problem is reformulated in a more macroscopic description where $g(\boldsymbol{x}, \boldsymbol{R})$ is replaced by its second moment $\boldsymbol{m}(\boldsymbol{x}, R)$ as it has been suggested by Phan-Thien in Phan-Thien (1995). Let $\boldsymbol{n}$ be the unit vector

$\boldsymbol{n}=\frac{\boldsymbol{R}}{R}$

where $R=|\boldsymbol{R}|$, the second moment writes:

$m_{\alpha \beta}=\frac{R^{2}}{4 \pi} \int_{\mathcal{S}}\left(n_{\alpha} n_{\beta} g\right) \mathrm{d} \boldsymbol{n}$

with $\mathcal{S}$ the surface of sphere of radius $R$.

However, the equation that governs the evolution of the second-order moment involves the fourth order and so on. Thus, a closure relation relating the fourth-order moment as a function of the lower-order moments is compulsory. This relation constitutes the so-called closure relation.

The contribution of this paper is to solve numerically the pair correlation function without reducing it to its second moment avoiding by the way the use of any closure relation needed to solve the equation governing the evolution of the second moment $\boldsymbol{m}(\boldsymbol{x}, R)$ (see Zmievski et al. 2005). We shall not contribute in any way to the formulation of micro-hydrodynamic suspensions model: the pair correlation function is taken as a micro-macro model and is solved using advanced model reduction techniques based on a separated representation, the so-called Proper Generalized Decomposition (PGD) that works efficiently in high dimensional spaces by circumventing the curse of dimensionality that such models imply (cf. "Tensor form of the discrete problem" section and "Appendix 2").

\section{Governing equations of the model}

\section{Smoluchowski's equation}

Following Smoluchowski (1915), we describe the microstructure of the suspension by the pair correlation function $g(\boldsymbol{x}, \boldsymbol{R})$.

The suspensions under consideration are assumed to be semi-dilute, isothermal, monodisperse, and incompressible. The suspended particles are rigid spheres, all identical, with radius $a \ll L$, where $L$ is the characteristic length of the fluid container. Both $a$ and $L$ are assumed to be constants. The pair correlation function is highly influenced by the flow kinematics, i.e., influenced by both the type and the strength of the flow. Thus, we allow the suspensions to be spatially inhomogeneous in the sense that the pair correlation function $g$ is a function of $\boldsymbol{R}$ and also of $\boldsymbol{x}$, but we assume that the density $n_{\mathrm{p}}$ of the suspended spheres remains a constant independent of both $\boldsymbol{x}$ and the time $t$. It is well-known (see, e.g., Morris (2009) and Miller et al. (2009)) that in flows with varying shear rate, the spheres migrate and thus $n_{\mathrm{p}}$ becomes a function of both $\boldsymbol{x}$ and $t$. It is therefore necessary to adopt $n_{\mathrm{p}}(\boldsymbol{x}, t)$ as an additional state variable joining the velocity $\boldsymbol{v}(\boldsymbol{x}, t)$ and the pair correlation function $g(\boldsymbol{x}, \boldsymbol{R}, t)$. We intend to discuss rigid sphere suspensions in such extended setting in a future paper. In this paper, we consider flows with constant shear rates (in "Steady-state solution in a simple shear flow" section) for which the extended setting is not necessary, but we also investigate spatially varying shear rates (in "Computing the pair correlation function in a simple recirculating flow" section) for which the setting with $n_{\mathrm{p}}=$ const . provides only an approximative model in which the migration of the spheres is ignored. How much will the results obtained in "Computing the pair correlation function in a simple recirculating flow" section change if the migration is 
taken into account remains an open question that will be addressed in future works.

The Smoluchowski equation (Smoluchowski 1915) (see also Zmievski et al. (2005) and references cited therein) writes:

$$
\begin{gathered}
\frac{\partial g}{\partial t}=-\frac{\partial}{\partial x_{\alpha}}\left(g v_{\alpha}\right)-\frac{\partial}{\partial R_{\alpha}}\left(g R_{\beta} \frac{\partial v_{\alpha}}{\partial R_{\beta}}\right)+\frac{\partial}{\partial R_{\gamma}}\left(g \lambda_{\gamma \alpha \beta} \boldsymbol{D}_{\alpha \beta}\right) \\
+\frac{\partial}{\partial R_{\alpha}}\left(g \boldsymbol{\Lambda}_{\alpha \beta} \frac{\partial}{\partial R_{\beta}}\left(U+k_{\mathrm{B}} T \ln g\right)\right)
\end{gathered}
$$

By $\boldsymbol{v}(\boldsymbol{x})$, we denote the overall velocity of the suspension and $\boldsymbol{D}$ denotes the rate of strain tensor,

$D_{\alpha \beta}=\frac{1}{2}\left(\frac{\partial v_{\alpha}}{\partial x_{\beta}}+\frac{\partial v_{\beta}}{\partial x_{\alpha}}\right)$

and the tensors $\boldsymbol{\Lambda}$ and $\lambda$ are kinetic coefficients which are functions of $\boldsymbol{R}$ (see "Appendix 1"). The total interparticle potential $U(\boldsymbol{R})$ writes:

$U=U_{\mathrm{HC}}+U_{\mathrm{nl}}$

with

$U_{\mathrm{HC}}(R)=\left\{\begin{array}{l}0, \text { for } R>2 a \\ \infty, \text { for } R \leq 2 a\end{array}\right.$

and

$U_{\mathrm{nl}}(R)=\frac{\epsilon}{R^{12}}$, for $R \geq 2 a$.

where $\epsilon$ is a positive constant. $g_{\text {eq }}$ denotes the equilibrium correlation function as

$g_{\text {eq }}(R)=\exp \left(\frac{U_{\mathrm{nl}}(R)}{k_{\mathrm{B}} T}\right)$

Equation 4 is subjected to two boundary conditions:

- When $R \rightarrow \infty, g(\boldsymbol{x}, \boldsymbol{R}) \rightarrow n_{\mathrm{p}}^{2}$.

- When $R=2 a$, the flux through the boundary vanishes.

According to Zmievski et al. (2005), the dimensionless form of the equation governing the evolution of the correlation function writes:

$$
\begin{aligned}
\frac{\partial g}{\partial t}= & -\operatorname{Pe} \frac{\partial}{\partial x_{\alpha}}\left(g v_{\alpha}\right)-\operatorname{Pe} \frac{\partial}{\partial R_{\alpha}}\left(R_{\beta} \frac{\partial v_{\alpha}}{\partial x_{\beta}} \Delta g\right) \\
& +\operatorname{Pe} \frac{\partial}{\partial R_{\gamma}}\left(g \lambda_{\gamma \alpha \beta} D_{\alpha \beta}\right) \\
& +\frac{\partial}{\partial R_{\alpha}}\left(g_{\text {eq }} \Lambda_{\alpha \beta} \frac{\partial}{\partial R_{\beta}}\left(\frac{\partial g}{\partial g_{\text {eq }}}\right)\right)
\end{aligned}
$$

where

$$
\begin{aligned}
& \boldsymbol{x} \rightarrow \boldsymbol{x} / a, \quad \boldsymbol{R} \rightarrow \boldsymbol{R} / a \\
& g \rightarrow \frac{g}{n_{\mathrm{p}}^{2}}, \quad U_{\mathrm{nl}} \rightarrow \frac{1}{k_{\mathrm{B}} T} U_{\mathrm{nl}}
\end{aligned}
$$

and the Péclet number defined as

$\mathrm{Pe}=\dot{\gamma} \frac{6 \pi \eta_{0} a^{3}}{k_{\mathrm{B}} T}$

with $\dot{\gamma}$ the strain rate and $\eta_{0}$ a positive coefficient. $\Delta g(R)=g(R)-g_{\text {eq }}(R)$ denotes the deviation from equilibrium of the correlation function.

For more details concerning definition of kinetic coefficients, please refer to "Appendix 1" as well as Zmievski et al. (2005) and the references therein.

\section{Expression of the stress tensor}

The stress tensor groups three contributions:

$\sigma=\sigma^{\text {hydr }}+\sigma^{\mathrm{DI}}+\sigma^{\mathrm{HI}}$

$\boldsymbol{\sigma}^{\text {hydr }}$ is the contribution from the Newtonian fluid in which particles are suspended. $\sigma^{\text {DI }}$ comes from the direct interactions including hard core and long-range potential. $\sigma^{\mathrm{HI}}$ is a contribution arising due to the hydrodynamic interactions.

The resulting dimensionless form of the extra stress tensor reads (Zmievski et al. 2005)

$$
\begin{aligned}
\sigma_{\alpha \beta}= & -\left(1+\frac{2}{5} \phi\right) D_{\alpha \beta}-\frac{27}{8 \pi} \frac{\phi^{2}}{\mathrm{Pe}} \\
& \times\left\{2 \int_{S_{2}} \mathrm{~d} \boldsymbol{n}\left(n_{\alpha} n_{\beta}-\frac{1}{3} \delta_{\alpha \beta}\right) \times\left[1-\mu_{1}(2)\right] \Delta g(2 \boldsymbol{n})\right. \\
& -\int \mathrm{d} \boldsymbol{R}\left[3\left(\mu_{1}-\mu_{2}\right)+R \frac{\partial \mu_{1}}{\partial \boldsymbol{R}}+\left(1-\mu_{1}\right) R \frac{\partial U_{\mathrm{nl}}}{\partial \boldsymbol{R}}\right] \\
& \left.\times\left(n_{\alpha} n_{\beta}-\frac{1}{3} \delta_{\alpha \beta}\right) \Delta g(\boldsymbol{R})\right\}
\end{aligned}
$$

where $\phi=(4 / 3) n_{\mathrm{p}} \pi a^{3}$ is the volume fraction of particles in the suspension and $\int_{S_{2}}$ refers to integration over the unit sphere $S_{2}$.

\section{Tensor form of the discrete problem}

The evolution equation of the pair correlation function involves both the physical and the configurational coordinates which are up to three dimensions each. If we account for the time, the total dimension of the problem is up to seven. In such high-dimensional spaces, the classical Finite Element (FE) method cannot be applied successfully because mesh-based discretization techniques suffer the so-called curse of dimensionality. 
The separated representation involved in the PGD (Ammar et al. 2006, 2007, 2009) allows circumventing or at least alleviating the curse of dimensionality encountered in the solution of the equation governing the evolution of the pair correlation function. The main idea of the PGD method is to find a solution of the linear system defined by

$\mathcal{A G}=\mathcal{B}$

where $\mathcal{A}, \mathcal{G}$, and $\mathcal{B}$ are written under separated form as a sum of tensorial products defined on a subspace of the initial domain. Without loss of generality, when choosing to separate the problem onto $D$ subspaces, $\mathcal{A}$, $\mathcal{G}$, and $\mathcal{B}$ write, respectively

$\mathcal{A}=\sum_{i=1}^{n_{A}} \mathbb{A}_{1}^{i} \otimes \mathbb{A}_{2}^{i} \otimes \ldots \otimes \mathbb{A}_{D}^{i}$

$\mathcal{G}=\sum_{i=1}^{n} \mathbb{G}_{1}^{i} \otimes \mathbb{G}_{2}^{i} \otimes \ldots \otimes \mathbb{G}_{D}^{i}$

$\mathcal{B}=\sum_{i=1}^{n_{B}} \mathbb{B}_{1}^{i} \otimes \mathbb{B}_{2}^{i} \otimes \ldots \otimes \mathbb{B}_{D}^{i}$

where $\mathcal{G}$ is the discrete tensor form of $g$. The PGD method is an a priori method, opposed to the a posteriori method (as the well-known Proper Orthogonal Decomposition method (POD)) and does not need information concerning the solution to compute the reduced approximation basis. Furthermore, PGD method can be used with any partial differential equation, written in continuous or discrete form, as long as the operator $\mathcal{A}$ and the right-hand side term $\mathcal{B}$ may be written under separated form. For the pair correlation function equation, numerous separation strategies are feasible, for example:

- the functions may be defined on subspaces of dimension 1 only, such as

$$
\mathcal{G}=\sum_{i=1}^{n} \mathbb{G}_{x}^{i} \otimes \mathbb{G}_{y}^{i} \otimes \mathbb{G}_{z}^{i} \otimes \mathbb{G}_{u}^{i} \otimes \mathbb{G}_{v}^{i} \otimes \mathbb{G}_{w}^{i} \otimes \mathbb{G}_{t}^{i}
$$

where time coordinate is $t$, space coordinates are $\boldsymbol{x}=(x, y, z)^{T}$, and configurational coordinates $\boldsymbol{R}=$ $(u, v, w)^{T}$,

- it is possible to separate the solution over physical, configurational, and time spaces

$$
\mathcal{G}=\sum_{i=1}^{n} \mathbb{G}_{\boldsymbol{x}}^{i} \otimes \mathbb{G}_{\boldsymbol{R}}^{i} \otimes \mathbb{G}_{t}^{i}
$$

- or isolate the physical and configurational spaces with a classical time discretization

$$
\mathcal{G}^{t}=\sum_{i=1}^{n} \mathbb{G}_{\boldsymbol{x}}^{i} \otimes \mathbb{G}_{\boldsymbol{R}}^{i}
$$

where $\mathcal{G}^{t}$ corresponds to the solution $\mathcal{G}$ at time $t$.

We chose to separate the problem on two subspaces, the physical and configurational spaces, and to treat the time space using classical discretization (an implicit Euler scheme) because it is the most convenient way to deal with the influence induced by the presence of the particles on the fluid evolution.

Let introduce the tensor form related to the Eq. 10 which can be rewritten as:

$$
\begin{aligned}
\frac{\partial g}{\partial t} & +\boldsymbol{v} \cdot \frac{\partial g}{\partial \boldsymbol{x}}+E_{0} g+\frac{\partial}{\partial \boldsymbol{R}}\left(\boldsymbol{E}_{1} g\right) \\
& -\frac{\partial}{\partial \boldsymbol{R}}\left(\boldsymbol{E}_{2} \frac{\partial}{\partial \boldsymbol{R}}\left(\frac{g}{g_{\text {eq }}}\right)\right)=F
\end{aligned}
$$

where

$$
\begin{aligned}
E_{0}(\boldsymbol{x}, \boldsymbol{R})= & \operatorname{Pe}\left(\frac{\partial v_{\gamma}}{\partial x_{\gamma}}+\frac{\partial R_{\beta}}{\partial R_{\alpha}} \frac{\partial v_{\alpha}}{\partial x_{\beta}}\right) \\
& -\operatorname{Pe}\left(\frac{\partial \lambda_{\gamma \alpha \beta}}{\partial R_{\gamma}} D_{\alpha \beta}+\lambda_{\gamma \alpha \beta} \frac{\partial D_{\alpha \beta}}{\partial R_{\gamma}}\right) \\
\boldsymbol{E}_{1}(\boldsymbol{x}, \boldsymbol{R})= & \operatorname{Pe} R_{\beta} \frac{\partial v_{\alpha}}{\partial x_{\beta}}-\operatorname{Pe}\left(\lambda_{\gamma \alpha \beta} D_{\alpha \beta}\right) \\
\boldsymbol{E}_{2}(\boldsymbol{x})= & g_{\mathrm{eq}} \lambda_{\alpha \beta} \\
F(\boldsymbol{x}, \boldsymbol{R})= & \operatorname{Pe}\left(\frac{\partial R_{\beta}}{\partial R_{\alpha}} \frac{\partial v_{\alpha}}{\partial x_{\beta}} g_{\text {eq }}+R_{\beta} \frac{\partial v_{\alpha}}{\partial x_{\beta}} \frac{\partial g_{\text {eq }}}{\partial R_{\alpha}}\right)
\end{aligned}
$$

Some of the above coefficients involve both the physical and the configurational coordinates, but their dependence on the physical space lies only through the velocity gradient. To rewrite the previous coefficients in a separate form, we chose to express their contribution in the velocity gradient canonical basis (see "Appendix 3" for more details concerning the procedure).

Thus $E_{0}(\boldsymbol{x}, \boldsymbol{R})$ can be rewritten as:

$$
E_{0}(\boldsymbol{x}, \boldsymbol{R})=\frac{\partial v_{\alpha}}{\partial x_{\beta}}(\boldsymbol{x}) E_{0}^{\alpha \beta}(\boldsymbol{R})
$$


We follow the same procedure for the other coefficients $E_{1}(\boldsymbol{x}, \boldsymbol{R})$ and $F(\boldsymbol{x}, \boldsymbol{R})$ leading to the weak form of Eq. 16:

$$
\begin{aligned}
\int_{\Omega \times \mathbf{I}} g^{*}\left(\frac{\partial g}{\partial t}+\boldsymbol{v} \cdot \frac{\partial g}{\partial \boldsymbol{x}}+E_{0} g\right. \\
\left.+\frac{\partial}{\partial \boldsymbol{R}}\left(\boldsymbol{E}_{1} g\right)-\frac{\partial}{\partial \boldsymbol{R}}\left(\boldsymbol{E}_{2} \frac{\partial}{\partial \boldsymbol{R}}\left(\frac{g}{g_{\mathrm{eq}}}\right)\right)\right) \mathrm{d} \boldsymbol{x} \mathrm{d} \boldsymbol{R} \\
=\int_{\Omega \times \mathbf{I}} g^{*} F \mathrm{~d} \boldsymbol{x} \mathrm{d} \boldsymbol{R}
\end{aligned}
$$

where the variables $\boldsymbol{x} \in \Omega$ and $\boldsymbol{R} \in \mathbf{I}$ are, respectively, the position vectors of the physical and configurational space. In this formulation, the second derivative term is rewritten as follows:

$$
\begin{aligned}
\int_{\Omega \times \mathbf{I}} & g^{*} \frac{\partial}{\partial \boldsymbol{R}}\left(\boldsymbol{E}_{2} \frac{\partial}{\partial \boldsymbol{R}}\left(\frac{g}{g_{\text {eq }}}\right)\right) \mathrm{d} \boldsymbol{x} \mathrm{d} \boldsymbol{R} \\
= & \int_{\Omega \times \Gamma} g^{*} \boldsymbol{E}_{2} \frac{\partial}{\partial \boldsymbol{R}}\left(\frac{g}{g_{\text {eq }}}\right) \mathrm{d} \boldsymbol{x} \mathrm{d} \boldsymbol{R} \\
& -\int_{\Omega \times \mathbf{I}} \frac{\partial g^{*}}{\partial \boldsymbol{R}} \boldsymbol{E}_{2} \frac{\partial}{\partial \boldsymbol{R}}\left(\frac{g}{g_{\text {eq }}}\right) \mathrm{d} \boldsymbol{x} \mathrm{d} \boldsymbol{R}
\end{aligned}
$$

where $\int_{\Gamma}$ is the integration over the boundary of $\mathbf{I}$. The problem is subjected to two boundary conditions for $R \rightarrow 2$ and $R \rightarrow \infty$. For the far-field limit, $R \rightarrow \infty$, we assume that the pair correlation function tends to the uniform distribution, i.e., $g=1$. At the contact surface of a pair $(R \rightarrow 2)$, we adopt the boundary condition introduced by Batchelor (1977): a zero flux condition. It means that the flux vanishes on $R=2$ :

$n_{\alpha}\left[\operatorname{Pe}\left(-R_{\beta} \frac{\partial v_{\alpha}}{\partial x_{\beta}}+\lambda_{\gamma \alpha \beta} D_{\alpha \beta}\right) g+\boldsymbol{E}_{2} \frac{\partial}{\partial R_{\beta}}\left(\frac{g}{g_{\text {eq }}}\right)\right]=0$

with $n_{\alpha}=R_{\alpha} / R$. Thus, on the boundary $R=2$ :

$n_{\alpha}\left[\boldsymbol{E}_{2} \frac{\partial}{\partial R_{\beta}}\left(\frac{g}{g_{\text {eq }}}\right)\right]=n_{\alpha}[\underbrace{\operatorname{Pe}\left(R_{\beta} \frac{\partial v_{\alpha}}{\partial x_{\beta}}-\lambda_{\gamma \alpha \beta} D_{\alpha \beta}\right)}_{\boldsymbol{E}_{1}} g]$
Thus, the second derivative term writes:

$$
\begin{aligned}
\int_{\Omega \times \mathbf{I}} & g^{*} \frac{\partial}{\partial \boldsymbol{R}}\left(\boldsymbol{E}_{2} \frac{\partial}{\partial \boldsymbol{R}}\left(\frac{g}{g_{\text {eq }}}\right)\right) \mathrm{d} \boldsymbol{x} \mathrm{d} \boldsymbol{R} \\
= & \int_{\Omega \times \Gamma} g^{*}\left(\boldsymbol{n} \cdot \boldsymbol{E}_{1}\right) g \mathrm{~d} \boldsymbol{x} \mathrm{d} \boldsymbol{R} \\
& -\int_{\Omega \times \mathbf{I}} \frac{\partial g^{*}}{\partial \boldsymbol{R}} \boldsymbol{E}_{2}\left(\frac{1}{g_{\text {eq }}} \frac{\partial g}{\partial \boldsymbol{R}}+g \frac{\partial}{\partial \boldsymbol{R}}\left(\frac{1}{g_{\text {eq }}}\right)\right) \mathrm{d} \boldsymbol{x} \mathrm{d} \boldsymbol{R}
\end{aligned}
$$

Finally, the weak form reads:

$$
\begin{aligned}
& \int_{\Omega \times \mathbf{I}}\left(g^{*} \frac{\partial g}{\partial t}+g^{*} \boldsymbol{v} \cdot \frac{\partial g}{\partial \boldsymbol{x}}+g^{*} E_{0} g\right. \\
& \left.+g^{*} \boldsymbol{E}_{1} \frac{\partial g}{\partial \boldsymbol{R}}+\frac{\partial g^{*}}{\partial \boldsymbol{R}} \boldsymbol{E}_{2} \frac{\partial g}{\partial \boldsymbol{R}}+\frac{\partial g^{*}}{\partial \boldsymbol{R}} \boldsymbol{E}_{3} g\right) \mathrm{d} \boldsymbol{x} \mathrm{d} \boldsymbol{R} \\
& -\int_{\Omega \times \Gamma} g^{*}\left(\boldsymbol{n} \cdot \boldsymbol{E}_{1}\right) g \mathrm{~d} \boldsymbol{x} \mathrm{d} \boldsymbol{R}=\int_{\Omega \times \mathbf{I}} g^{*} F \mathrm{~d} \boldsymbol{x} \mathrm{d} \boldsymbol{R}
\end{aligned}
$$

with

$$
\begin{aligned}
E_{0}(\boldsymbol{x}, \boldsymbol{R})= & \operatorname{Pe}\left(\frac{\partial v_{\gamma}}{\partial x_{\gamma}}+\frac{\partial R_{\beta}}{\partial R_{\alpha}} \frac{\partial v_{\alpha}}{\partial x_{\beta}}\right) \\
& -\operatorname{Pe}\left(\frac{\partial \lambda_{\gamma \alpha \beta}}{\partial R_{\gamma}} D_{\alpha \beta}+\lambda_{\gamma \alpha \beta} \frac{\partial D_{\alpha \beta}}{\partial R_{\gamma}}\right) \\
\boldsymbol{E}_{1}(\boldsymbol{x}, \boldsymbol{R})= & \operatorname{Pe}\left(R_{\beta} \frac{\partial v_{\alpha}}{\partial x_{\beta}}-\lambda_{\gamma \alpha \beta} D_{\alpha \beta}\right) \\
\boldsymbol{E}_{2}(\boldsymbol{R})= & \Lambda_{\alpha \beta} \\
\boldsymbol{E}_{3}(\boldsymbol{R})= & \Lambda_{\alpha \beta} g_{\mathrm{eq}} \frac{\partial}{\partial \boldsymbol{R}}\left(\frac{1}{g_{\mathrm{eq}}}\right) \\
F(\boldsymbol{x}, \boldsymbol{R})= & \operatorname{Pe}\left(\frac{\partial R_{\beta}}{\partial R_{\alpha}} \frac{\partial v_{\alpha}}{\partial x_{\beta}} g_{\mathrm{eq}}+R_{\beta} \frac{\partial}{\partial R_{\alpha}}\left(\frac{\partial v_{\alpha}}{\partial x_{\beta}}\right) g_{\mathrm{eq}}\right. \\
& \left.+R_{\beta} \frac{\partial v_{\alpha}}{\partial x_{\beta}} \frac{\partial g_{\mathrm{eq}}}{\partial R_{\alpha}}\right)
\end{aligned}
$$

Equation 23 is discretized using a standard Finite Element approximation in each coordinate. In homogeneous (rheometric) flows, no specific strategy is needed to solve the pair correlation function. However, in complex flows (i.e., when the velocity gradient is no longer homogeneous), we use reduced modeling to alleviate difficulty related to the high-dimensional domain of definition of the pair correlation function. For this purpose, we introduce $\mathbf{N}$ and $\mathbf{M}$ that represent the vectors containing the shape functions associated, respectively, with each coordinate $\boldsymbol{x}$ and $\boldsymbol{R}$. 
By defining the following matrices:

$$
\begin{array}{rlrl}
\mathbb{A}_{\boldsymbol{x}}^{1} & =\int_{\Omega} \mathbf{N} \boldsymbol{v} \frac{\mathrm{d} \mathbf{N}^{T}}{\mathrm{~d} \boldsymbol{x}} \mathrm{d} \boldsymbol{x} & \mathbb{A}_{\boldsymbol{R}}^{1} & =\int_{\mathbf{I}} \mathbf{M} \mathbf{M}^{T} \mathrm{~d} \boldsymbol{R} \\
\mathbb{A}_{\boldsymbol{x}}^{2}=\int_{\Omega} \mathbf{N} \mathbf{N}^{T} \mathrm{~d} \boldsymbol{x} & \mathbb{A}_{\boldsymbol{R}}^{2}=\int_{\mathbf{I}} \frac{\mathrm{d} \mathbf{M}}{\mathrm{d} \boldsymbol{R}} \boldsymbol{E}_{2} \frac{\mathrm{d} \mathbf{M}^{T}}{\mathrm{~d} \boldsymbol{R}} \mathrm{d} \boldsymbol{R} \\
\mathbb{A}_{\boldsymbol{x}}^{3}=\int_{\Omega} \mathbf{N} \mathbf{N}^{T} \mathrm{~d} \boldsymbol{x} & \mathbb{A}_{\boldsymbol{R}}^{3}=\int_{\mathbf{I}} \frac{\mathrm{d} \mathbf{M}}{\mathrm{d} \boldsymbol{R}} \boldsymbol{E}_{3} \mathbf{M}^{T} \mathrm{~d} \boldsymbol{R} \\
\mathbb{A}_{\boldsymbol{x}}^{3(\alpha-1)+\beta+3}=\int_{\Omega} \mathbf{N} \frac{\mathrm{d} v_{\alpha}}{\mathrm{d} x_{\beta}} \mathbf{N}^{T} \mathrm{~d} \boldsymbol{x} & \mathbb{A}_{\boldsymbol{R}}^{3(\alpha-1)+\beta+3}= & \int_{\mathbf{I}} \mathbf{M} E_{0}^{\alpha \beta} \mathbf{M}^{T}+\mathbf{M} \boldsymbol{E}_{1}^{\alpha \beta} \frac{\mathrm{d} \mathbf{M}^{T}}{\mathrm{~d} \boldsymbol{R}} \mathrm{d} \boldsymbol{R} \\
& -\int_{\Gamma} \mathbf{M}\left(\boldsymbol{n} \cdot \boldsymbol{E}_{1}^{\alpha \beta}\right) \mathbf{M}^{T} \mathrm{~d} \boldsymbol{R} \\
\mathbb{B}_{\boldsymbol{x}}^{3(\alpha-1)+\beta}=\int_{\Omega} \frac{\mathrm{d} v_{\alpha}}{\mathrm{d} x_{\beta}} \mathbf{N} \mathrm{d} \boldsymbol{x} & \mathbb{B}_{\boldsymbol{R}}^{3(\alpha-1)+\beta} & =\int_{\mathbf{I}} F^{\alpha \beta} \mathbf{M} \mathrm{d} \boldsymbol{R}
\end{array}
$$

the discrete form results:

$\mathcal{A G}=\mathcal{B}$

with

$\mathcal{A}=\sum_{i=1}^{12} \mathbb{A}_{\boldsymbol{x}}^{i} \otimes \mathbb{A}_{\boldsymbol{R}}^{i}$

and

$\mathcal{B}=\sum_{i=1}^{9} \mathbb{B}_{\boldsymbol{x}}^{i} \otimes \mathbb{B}_{\boldsymbol{R}}^{i}$

Note that this equation is a tensorial equation. The unknown field $\mathcal{G}$ contains the tensor product of the two functions given in Eq. 15. Thus, the resulting problem becomes non-linear and an appropriate linearization technique must be applied (see "Appendix 2").

\section{Numerical results}

The pair correlation function is defined both on the physical space and configurational space, up to three dimensions each. In what follows, we choose to represent the configurational space either in two or three dimensions.
Steady-state solution in a simple shear flow

We solve the steady pair correlation function in a simple shear flow where the velocity gradient is defined by

$\nabla \boldsymbol{v}=\left[\begin{array}{lll}0 & 1 & 0 \\ 0 & 0 & 0 \\ 0 & 0 & 0\end{array}\right]$

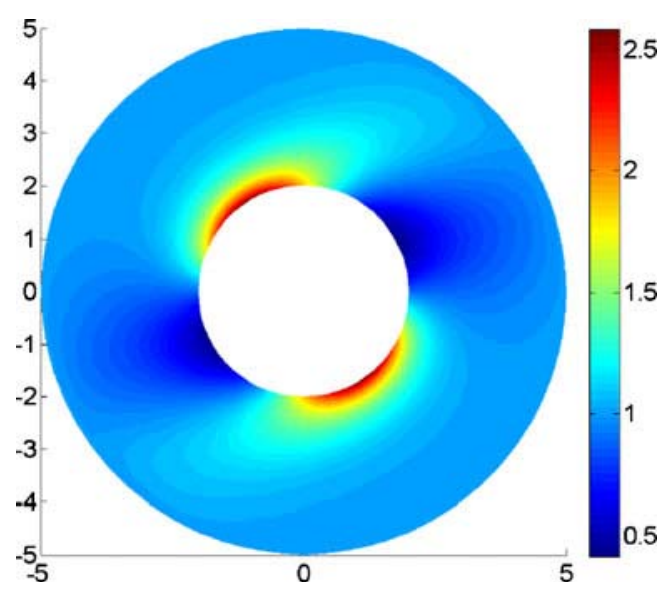

Fig. 1 Steady-state pair correlation function in simple shear flow in a 2-D configuration space. The Péclet number is set to 1 and $\epsilon=0$ 
By doing this, we can compare the results obtained with the ones given in Zmievski et al. (2005) and then evaluate the impact of the closure introduced in Zmievski et al. (2005). In this case, the physical space disappears and Eq. 18 is solved, which is a classical Finite Element formulation which does not require any special numerical strategy. According to Zmievski's results (see Zmievski et al. 2005), the suspension reach an equilibrium state for $R>5$. Thus, we choose to represent the configuration space by a hollow disk and a hollow sphere, respectively, in two and three dimensions, with an internal radius of 2 and the external one of 5 .

By solving the pair correlation function instead of reducing it to the second-order tensor $\boldsymbol{m}$, it is clear that we retain more information on the microstructure. We can see on Figs. 1 and 2 the orientation of the microstructure revealed by the angular dependence of $g$. We observe that the anisotropy decreases as $R$ increases, i.e., $g$ tends to 1 , which corresponds with the prescribed boundary condition $\left.g\right|_{R=5}=1$. On the other hand, for small $R$, the anisotropy of the microstructure is significant and orientated in the direction of the velocity gradient in perfect agreement with the Zmievski 's results (Zmievski et al. 2005). Notice in Figs. 1 and 2 the magnitude of the predicted anisotropy which is quite different when considering $2 \mathrm{D}$ or $3 \mathrm{D}$ configuration spaces whereas the distribution on the plane of shear is similar.

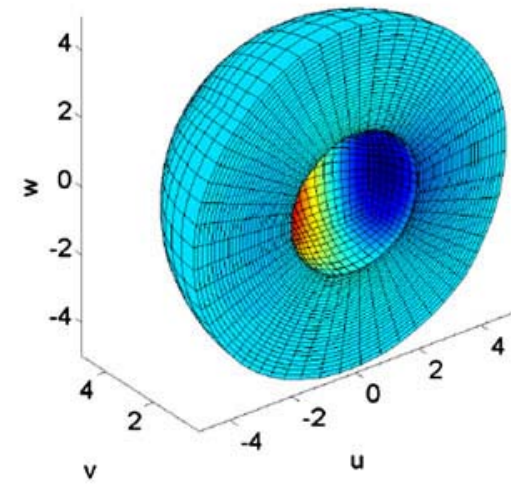

(a) $3 \mathrm{D}$

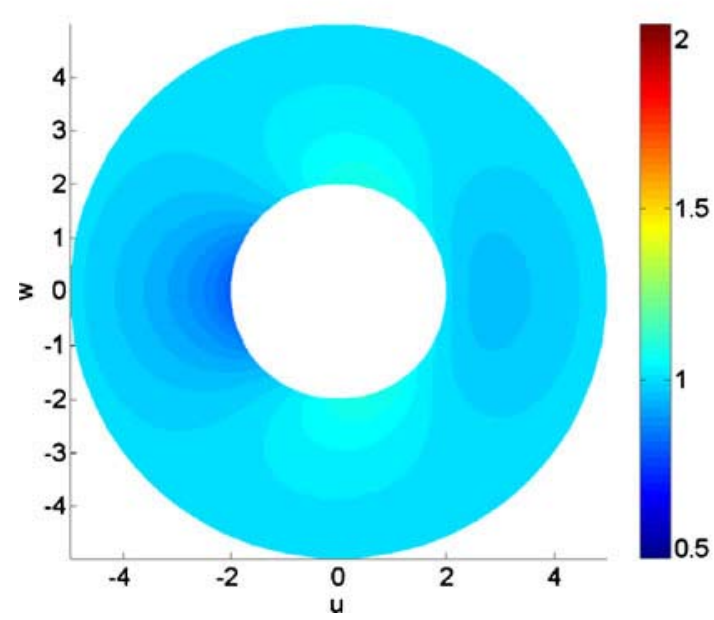

(c) $w$ vs $u$.
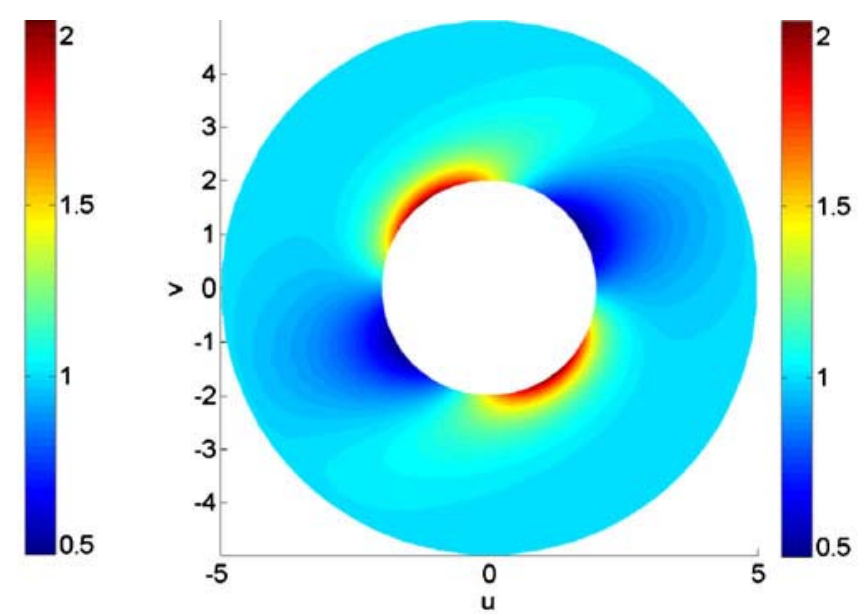

(b) $v$ vs $u$.

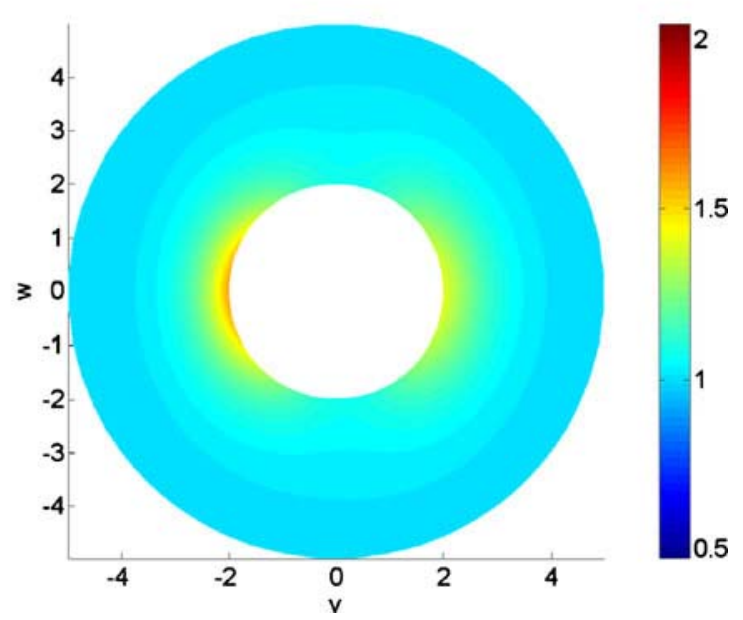

(d) $w$ vs $v$.

Fig. 2 Steady-state pair correlation function in simple shear flow in a 3-D configuration space with vector $\boldsymbol{R}=(u v w)^{T}$. The Péclet number is set to 1 and $\epsilon=0$ 


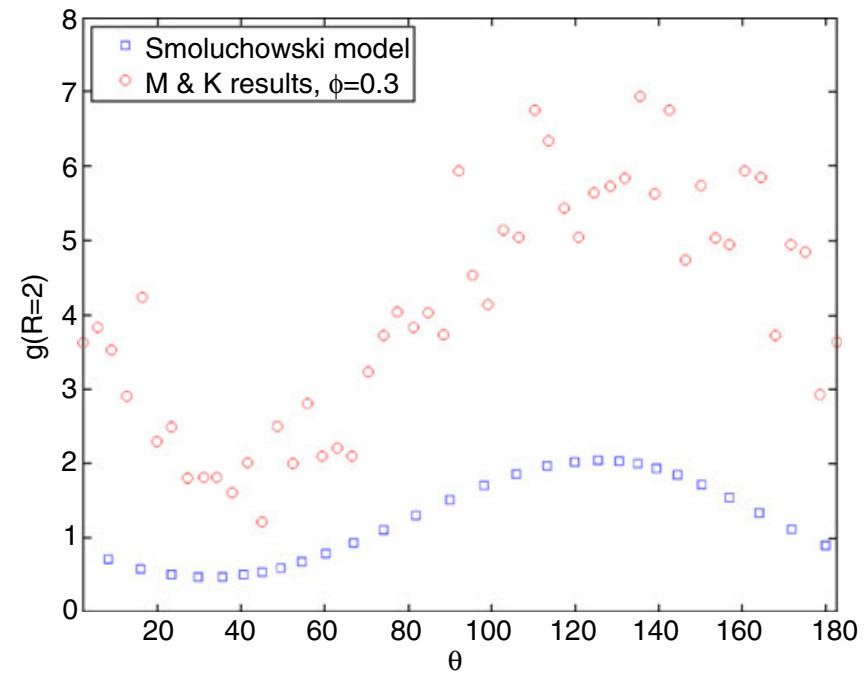

(a) $\mathrm{Pe}=1$.

Fig. 3 Pair correlation function for $R=2$ versus the angle on the plane of shear. Comparison with the results obtained by Morris and Katyal (2002) (noted M\&K) using Stokesian

Figure $3 \mathrm{a}, \mathrm{b}$ depicts the pair correlation function in a simple shear flow on the shear plane $(w=0)$. We represent $g(2, \theta)$ as a function of the angle in a cylindrical coordinate representation, in order to compare our predictions to the ones in Morris and Katyal (2002) (quoted $\mathrm{M} \& \mathrm{~K}$ ) that considered Stokesian dynamics. For $\mathrm{Pe}=1$, the maximum is located around $\theta=135^{\circ}$ and the minimum around $\theta=45^{\circ}$ that correspond to the compression and extension directions, respectively.

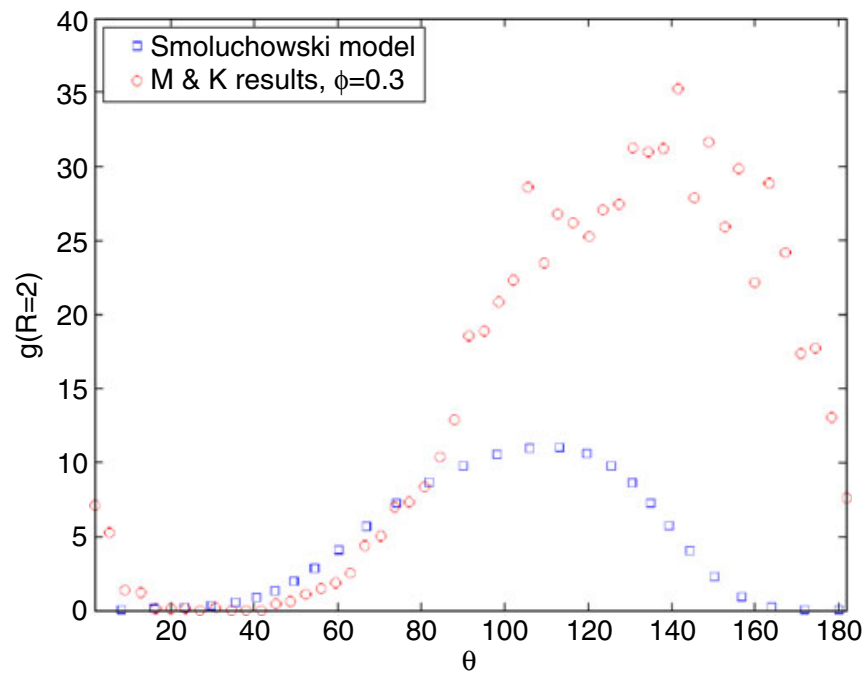

(b) $\mathrm{Pe}=25$.

dynamic approach, for $\phi=0.3$ and $\mathrm{Pe}=1$ and $\mathrm{Pe}=25$, and Smoluchowski model with $\epsilon=0$ and a 3D configurational space

These observations are in perfect agreement with the analysis (Batchelor 1977), and they agree with the orientation predicted by M\&K. For $\mathrm{Pe}=25$ the maximum is slightly shifted, and its is reached around $\theta=120^{\circ}$, always in the compression quadrant, but showing a slight difference with respect to the predictions of M\&K. The maximum amplitude obtained for $\mathrm{Pe}=1$ and $\mathrm{Pe}=25$ is $g(2) \approx 2$ and $g(2) \approx 11$ respectively, and it shows a noticeable difference with

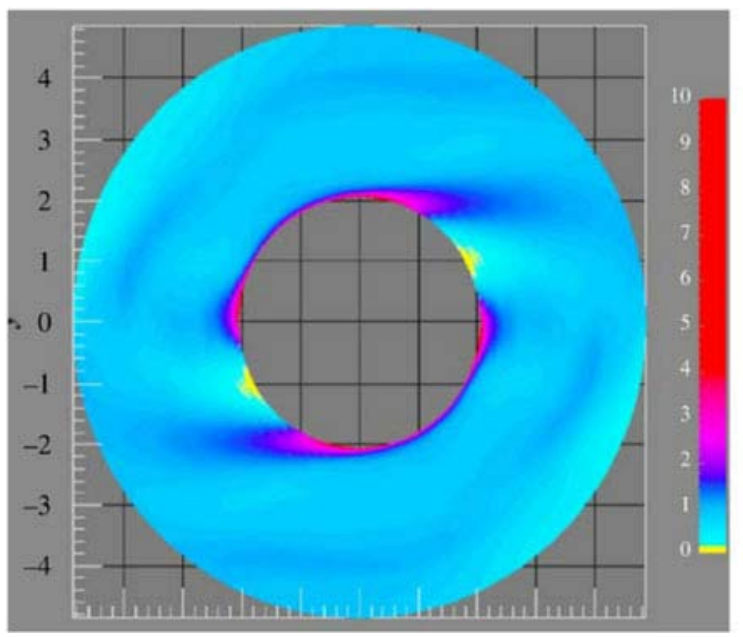

(a) Stokesian Dynamics by [21] $\phi=0.3, P e=25$.

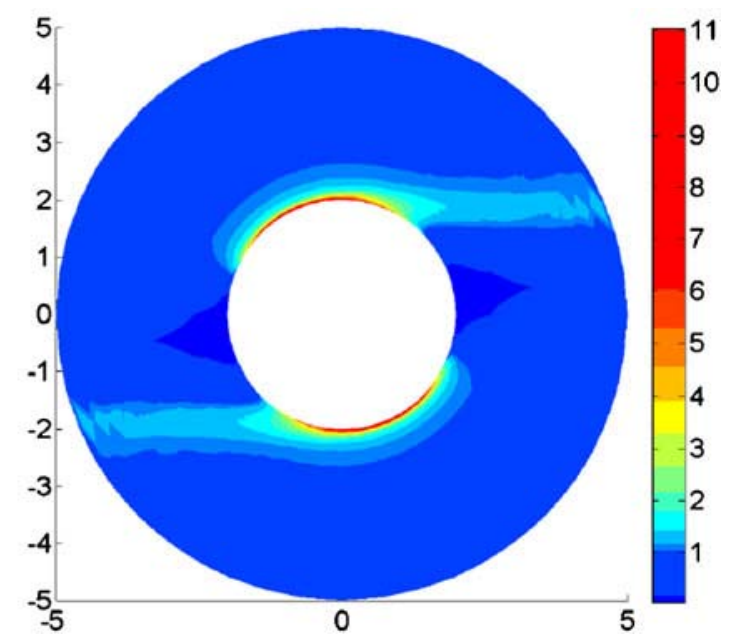

(b) Smoluchowski model, $P e=25, \epsilon=0$.

Fig. $4 g(\boldsymbol{R})$ on the shear plane for the results obtained by Morris and Katyal (2002) (a) and with the Smoluchowski model (b) with a 3D configurational space 


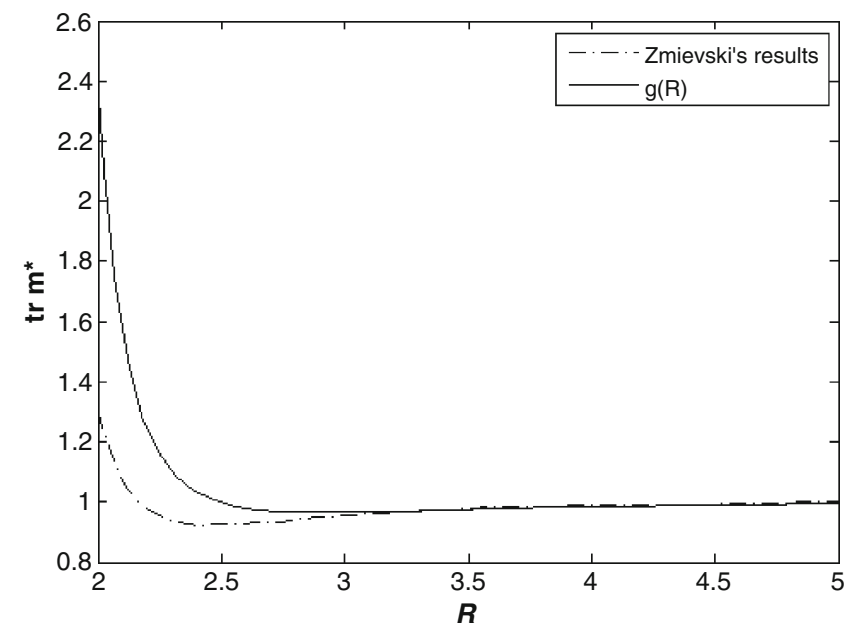

Fig. 5 Steady-state value of the trace of the dimensionless microstructure tensor, $\operatorname{tr} \boldsymbol{m}^{*}$, in a simple shear flow with $\mathrm{Pe}=10$ and $\epsilon=0$

respect to the $M \& K$ predictions, but it agrees with the theoretical results in Brady and Morris (1997) for suspensions of rigid spheres whose predictions scales as $\mathcal{O}\left(\mathrm{Pe}^{0.78}\right)$.

Figure $4 \mathrm{a}, \mathrm{b}$ depicts $g(\boldsymbol{R})$ on the shear plane in a simple shear flow for $\mathrm{Pe}=25$. Figure 4 is extracted from Morris (2009) and is obtained with Stokesian dynamic calculations with $\phi=0.3$ whereas Fig. 4b is obtained by solving the Smoluchowski model with $\epsilon=0$. Though the maximum magnitude is different (as seen previously) and there is a small difference in the

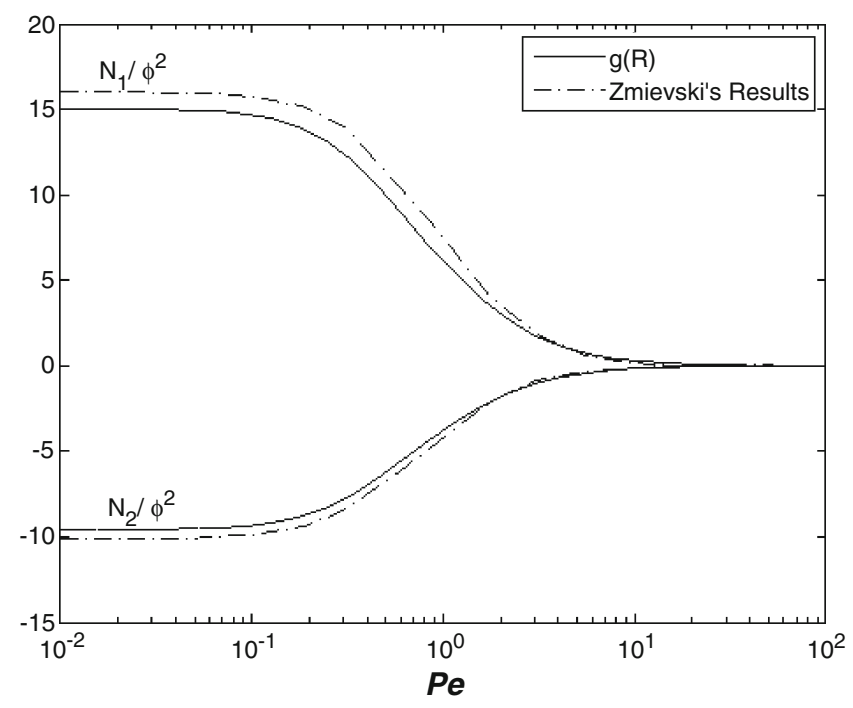

Fig. 6 Steady-state values of the dimensionless first $\left(N_{1}\right)$ and second $\left(N_{2}\right)$ normal stress coefficient, versus Péclet number Pe in a simple shear flow of colloidal suspension with $\epsilon=0$

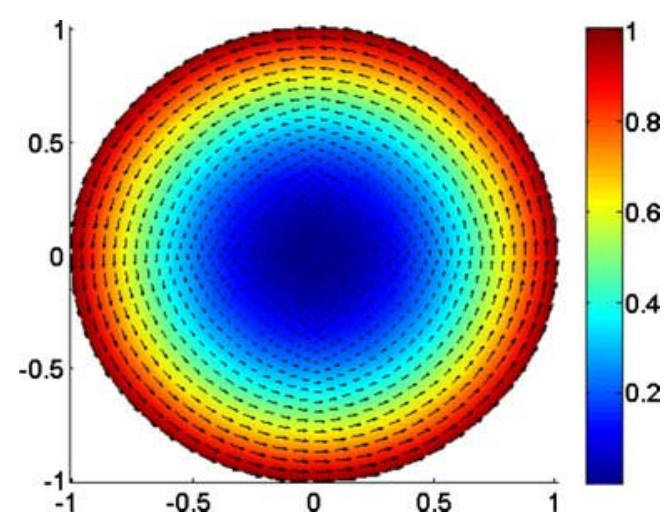

Fig. 7 Geometry and velocity field in physical space

orientation of the distribution, we can notice a quite good agreement between both results.

Moreover, by extracting the second moment tensor $\boldsymbol{m}$ from the pair correlation distribution function $g$ and comparing to Zmievski's results (Figs. 5 and 6), we see that the introduction of a closure relation influences strongly the solution in a simple shear flow (with $\mathrm{Pe}=$ 10 and $\epsilon=0$ ). By comparing the dimensionless first and second stress coefficient,

$N_{1}=\operatorname{Pe}^{-1}\left(\sigma_{22}-\sigma_{11}\right)$

and

$N_{2}=\operatorname{Pe}^{-1}\left(\sigma_{33}-\sigma_{22}\right)$

with $\sigma_{\alpha \beta}$ the components of the dimensionless extrastress tensor, we can notice the error induced by the closure relation used by Zmievski (2005) for calculating the microstructure tensor.

From a physical point of view, the thinning behavior observed for the first normal stress coefficient is in accordance with experimental measurements (Kolli

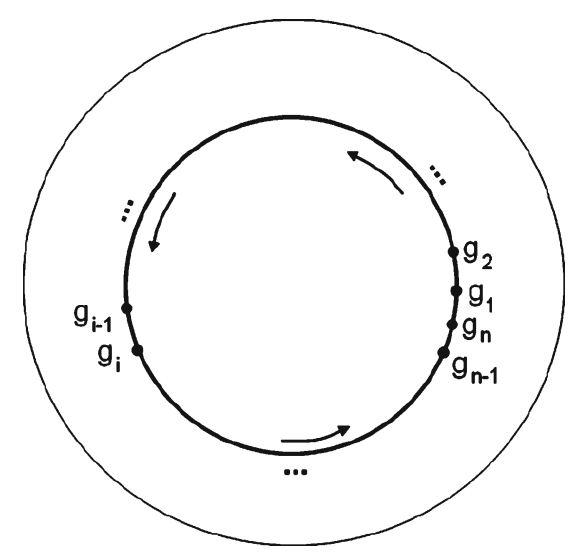

Fig. 8 Evolution of $g$ along a trajectory for FE calculation 
et al. 2002; Ohl and Gleissle 1993; So et al. 2001), as well as the negative value of the second stress coefficient. However, for large Pe, thickening behavior which has been observed experimentally (Kolli et al. 2002; Ohl and Gleissle 1993; So et al. 2001) and numerically (Brady 2001; Foss and Brady 2000) in concentrated suspensions cannot be reproduced by the model here considered.

Computing the pair correlation function in a simple recirculating flow

We compute the evolution of the pair correlation function in the recirculating flow depicted in Fig. 7. To evaluate the accuracy of the PGD solution, we compare it with the one computed by applying the Finite Element method.

The flow is defined by the velocity field

$\boldsymbol{v}=\left(\begin{array}{c}-y \sqrt{x^{2}+y^{2}} \\ x \sqrt{x^{2}+y^{2}}\end{array}\right)$.

Because Finite Element calculations fail when considering the whole physical and conformational domains, we adopt the following strategy: $g$ is computed only along a streamline (which is a perfect circle in the flow here addressed) taking into account that the equation governing the pair correlation function $g$ is purely advective in the physical space. The steady-state solution is then computed from an isotropic state after several rotations (cf. Fig. 8).

Note that the FE solution is computed at a single position (in the present case, it suffices because the problem symmetry) whereas the PGD solution is computed for the whole physical space.

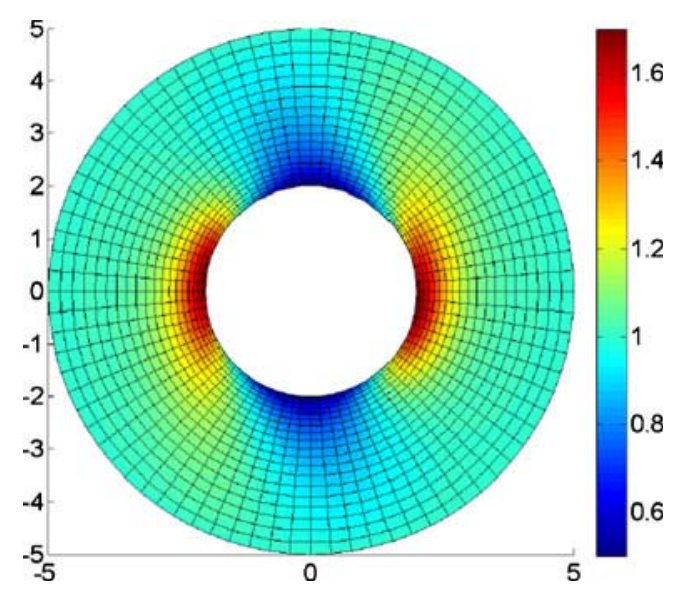

Fig. 9 Configurational space obtained with FE method

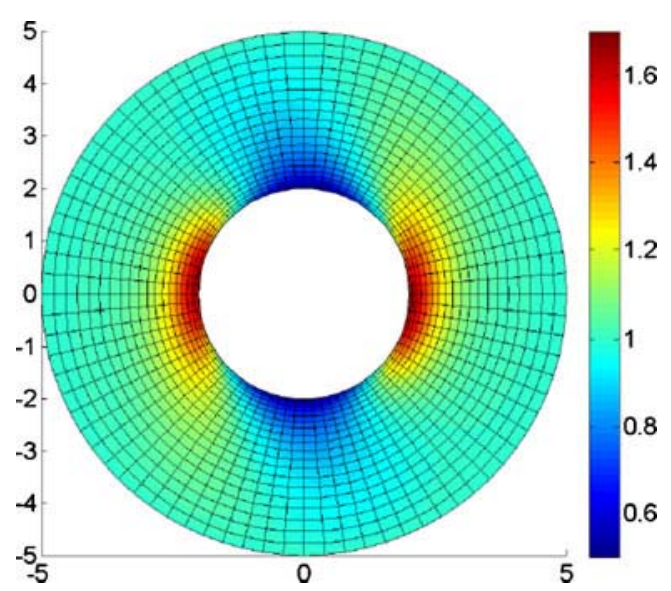

Fig. 10 Configurational space obtained with PGD method after 50 iterations

The problem is discretized by considering 3,905 and 1,680 degrees of freedom, respectively, for the physical (two-dimensional) and conformational (twodimensional) domains. Figures 9 and 10 depict the results obtained, respectively, with the FE and PGD method after 50 enrichments (that is, the PGD solution consists in 50 modes).

As it can be noticed, the results shown in Figs. 9 and 10 are very close, and we need to reduce the pair correlation function to its second-order moment tensor in order to appreciate the difference between FE and PGD solutions.

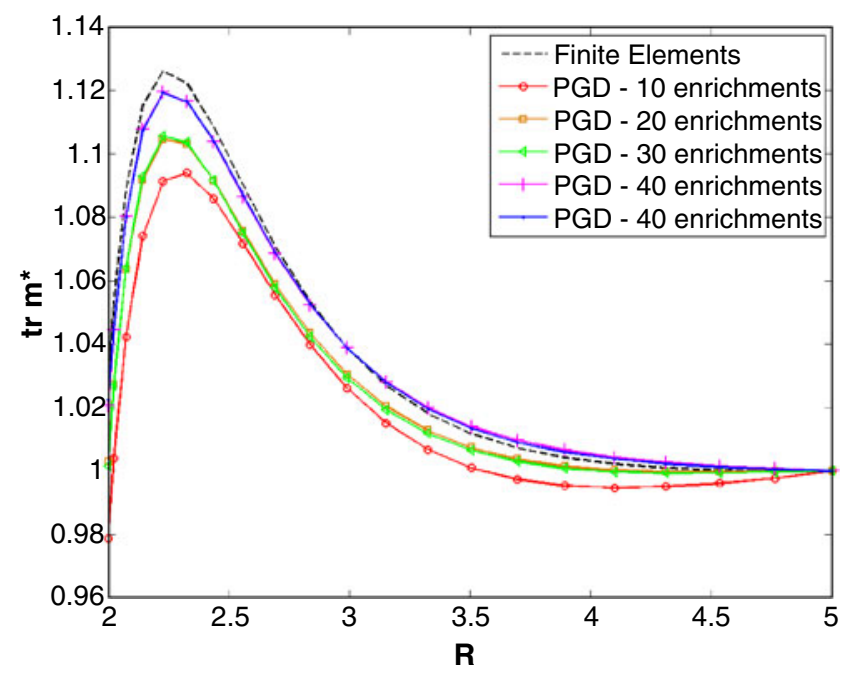

Fig. 11 Trace of the second-order moment of the pair correlation function computed at position $(-0.51,0.33)$ as a function of the number of terms involved in the separated representation of the pair correlation function 
Fig. 12 Five first functions involved in the PGD representation defined on the physical (top) and configurational space (bottom)
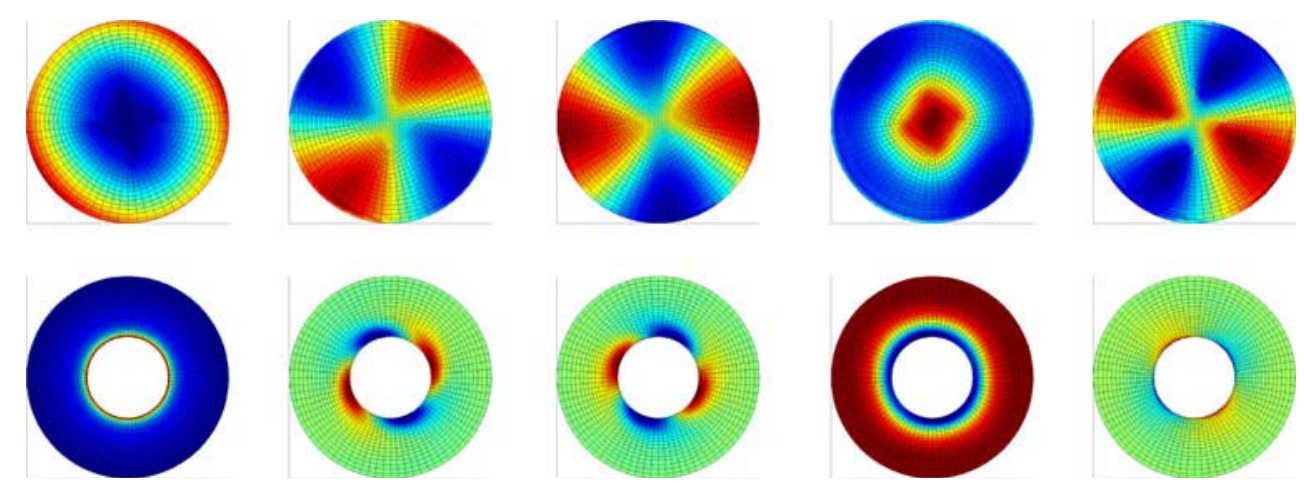

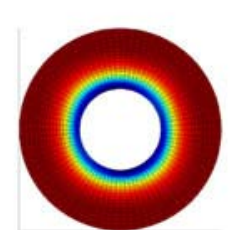

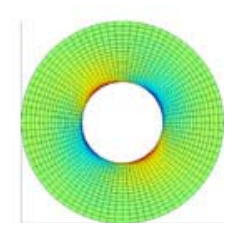

Figure 11 shows the trace of the second-order moment of the pair correlation function computed at position $(-0.51,0.33)$ as a function of the number of terms involved in the separated representation of the pair correlation function. The solution computed by applying the PGD method even with few enrichments (of around ten terms in the separated representation) is close to the reference FEM solution even if 40 enrichments are required to achieve the desired accuracy.

Figure 12 shows the five first functions defined on each subspace involved in the separated representation of the solution.

In this geometry, the fluid velocity field is assumed known, and we look for the steady state of the pair correlation function, allowing us to perform some numerical test illustrating the PGD capabilities. First, we represent in Fig. 13 the error of the PGD solution as

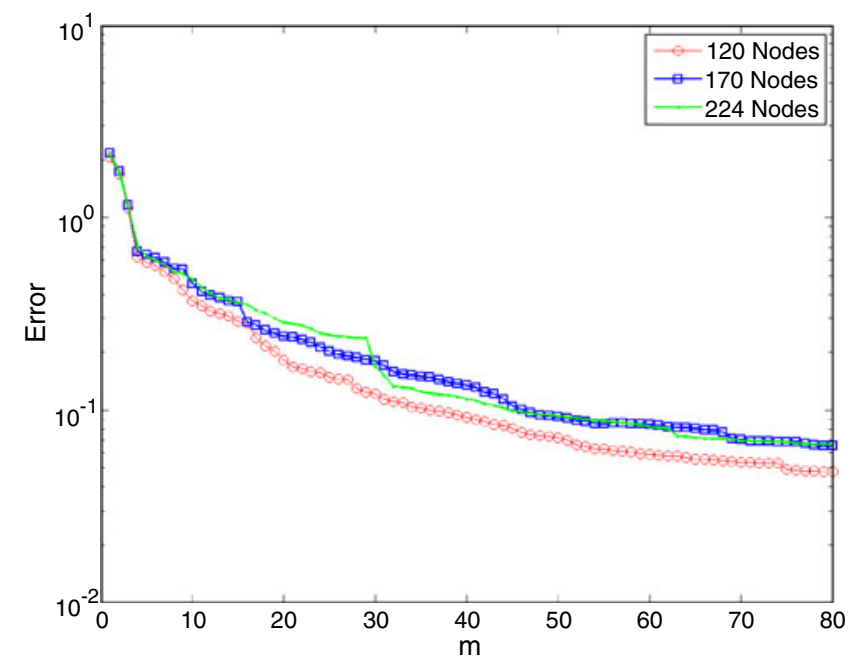

Fig. 13 Error of the PGD method as a function of the number of terms $m$ involved in the separated representation of the pair correlation function and for three different refinements of the configurational space a function of the number of enrichments $m$ for three different meshes.

The error is defined by

Err $=\left\|\operatorname{Sol}_{\mathrm{FE}}-\mathrm{Sol}_{\mathrm{PGD}}\right\|_{2}$

where Sol $_{\mathrm{FE}}$ and Sol $\mathrm{PGD}_{\mathrm{PD}}$ are, respectively, the solutions obtained with FE and PGD method.

The influence of the refinement on the convergence in not really significant as the error reached after 80 iterations remains almost unchanged, although the finer mesh has twice more nodes than the coarser one. The optimality of the separated representation, that is, the number of modes that it involves, constitutes a work in progress (Nouy 2010).

When plotting the calculation time of the FE and PGD method as a function of the total number of degrees of freedom (see Fig. 14), we can notice the efficiency of the PGD for treating high-dimensional

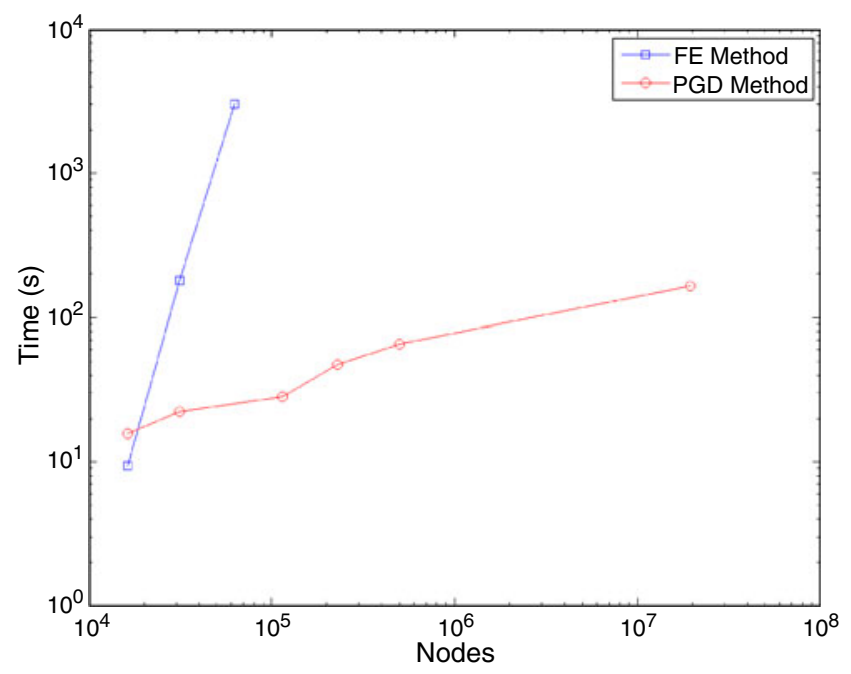

Fig. 14 Calculation time for both FE and PGD methods as a function of the total number of degrees of freedom used for describing the problem 
Fig. 15 Fluid velocity field and associated streamline after the first iteration

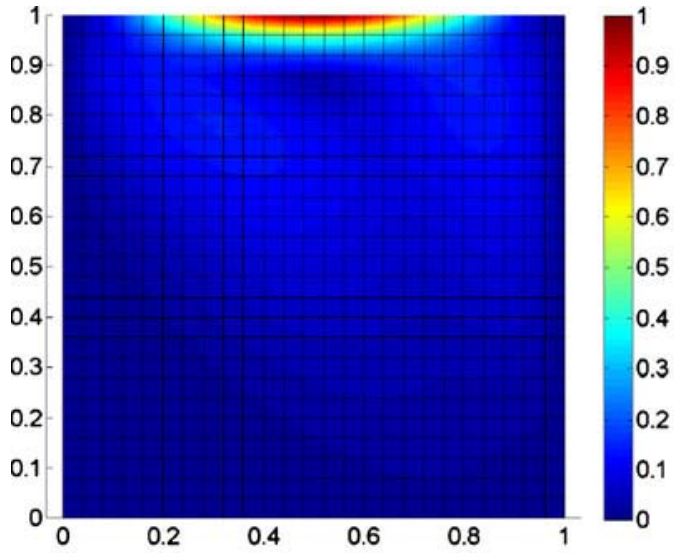

(a) Fluid velocity field.

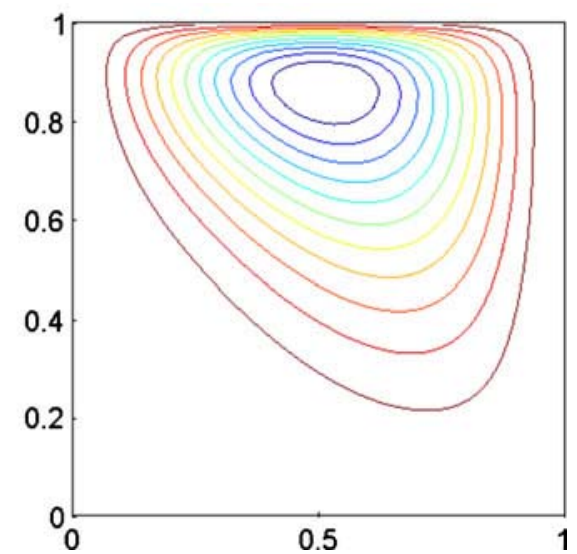

(b) Streamlines.

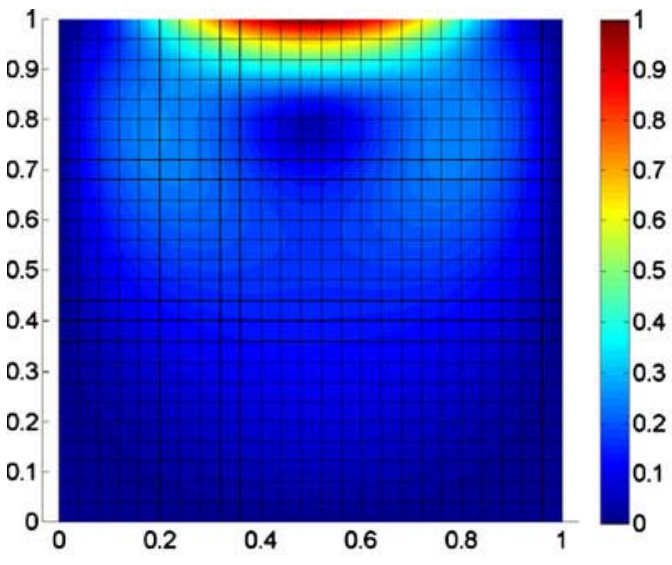

(a) Fluid velocity field.

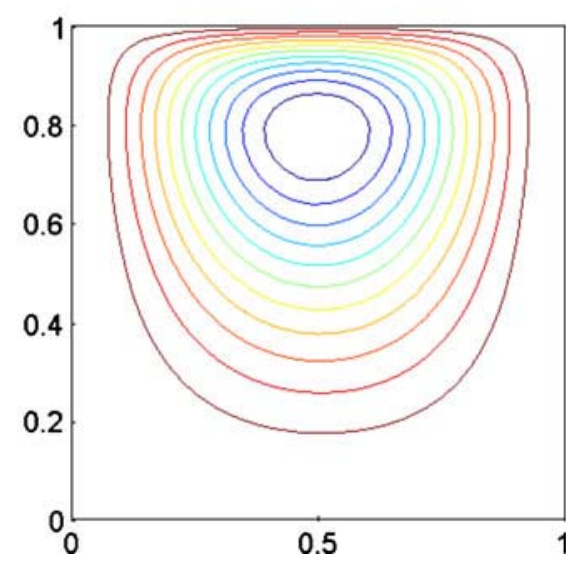

(b) Streamlines.
Fig. 16 Fluid velocity field and associated streamline at iteration 10
Fig. 17 Five first functions involved in the PGD separated representation defined on the physical (top) and configurational space (bottom)
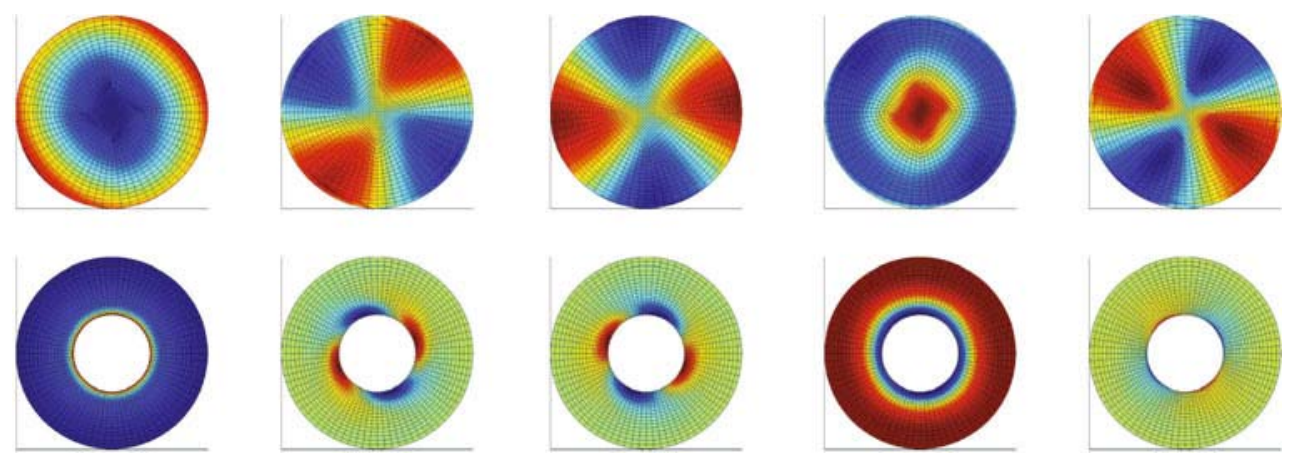


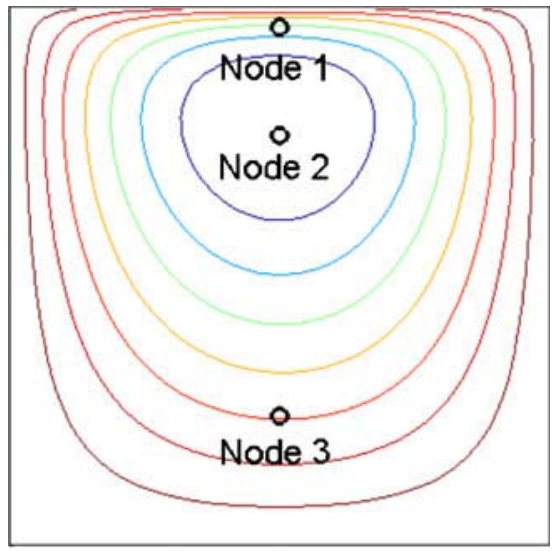

Fig. 18 Nodes considered for evaluating the pair correlation function

problem. These results have been obtained on a standard laptop with Intel Core i7 @ 2-GHz Processor with 6 GB of RAM.

Solving the pair correlation function in complex flows

For illustrating the performances of the PGD for addressing complex flows, in what follows we consider the driven cavity flow problem. We consider a suspension characterized by $\phi=0.05$ with long-range particle interactions $\left(\epsilon=10^{6}\right.$ in Eq. 8).

In addition to the equation governing the pair correlation function that we considered in the previous sections, now we should consider also the ones related to the fluid flow kinematics that involve the mass and momentum balance equations:

$\frac{\partial v_{\alpha}}{\partial t}=-\operatorname{Pe} \frac{\partial}{\partial x_{\beta}}\left(v_{\beta} u_{\alpha}+I_{\alpha \beta} p+\frac{1}{R e} \sigma_{\alpha \beta}\right)$

$\nabla \cdot \boldsymbol{v}=0$ where $\boldsymbol{u}=\boldsymbol{v} / \rho, \boldsymbol{\sigma}$ is defined in Eq. 11 and $R e$ the Reynolds number defined by

$R e=\frac{\rho a^{2} \dot{\gamma}}{\eta_{0}}$

The physical (2D) and the conformational (2D) domains are discretized by employing 1,976 and 1,680 nodes. At $t<0$, the fluid is at rest, i.e., $\boldsymbol{v}=\mathbf{0}$, and at $t=0$, we impose a velocity on the upper side defined by:

$v(x)=\frac{16}{h^{4}} v_{\max } x^{2}(h-x)^{2}$

with the width of the container $h=1$ and $v_{\max }=1$.

We perform the calculation for ten time steps with $\Delta t=0.01$. The resolution of the non-linear equation 37 is performed using a Newton-Raphson procedure, and 40 terms of the separated representation in the PGD framework were calculated at each time step (this number was enough for reaching the desired accuracy). For sake of simplicity in what follows, the word iteration refers to time step.

The fluid velocity field is depicted in Figs. 15 and 16, respectively, at iterations 1 and 10 .

Figure 17 shows the five first functions defined on each subspace involved in the separated representation of the solution $\mathcal{G}$ at iteration 10 .

We evaluate the evolution of the pair correlation function for the three different nodes in the physical space depicted in Fig. 18 with the corresponding velocity gradient:

$$
\begin{aligned}
& \nabla \boldsymbol{v}_{\text {node } 1}=\left(\begin{array}{cc}
0.22 & 6.79 \\
-0.53 & -0.22
\end{array}\right), \\
& \nabla \boldsymbol{v}_{\text {node } 2}=\left(\begin{array}{ll}
-0.01 & 1.60 \\
-1.30 & 0.01
\end{array}\right), \quad \nabla \boldsymbol{v}_{\text {node } 3}=\left(\begin{array}{rr}
-0.03 & -0.32 \\
-0.23 & 0.03
\end{array}\right)
\end{aligned}
$$

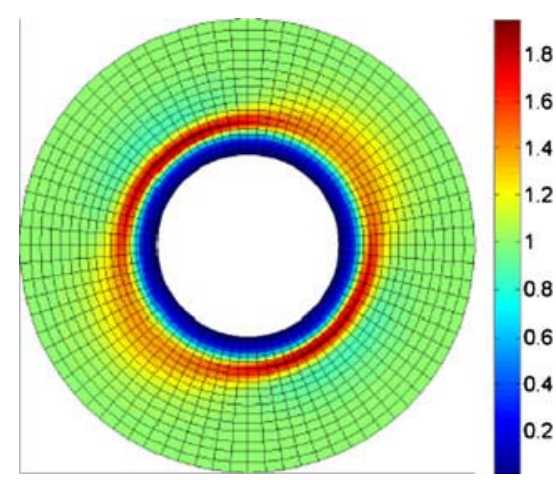

(a) Iteration 1.

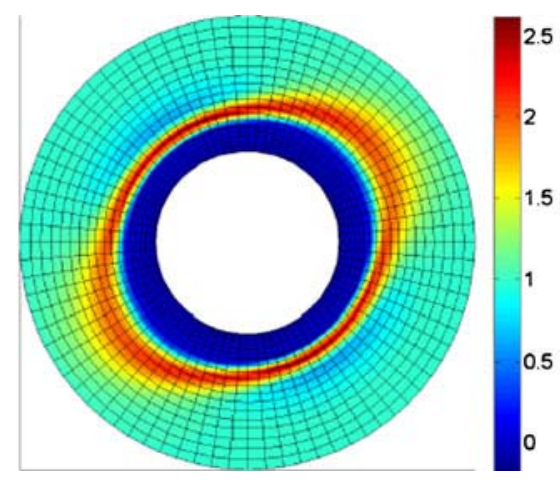

(b) Iteration 5 .

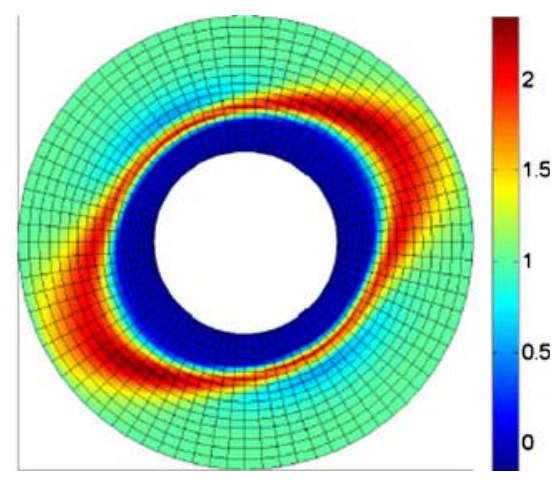

(c) Iteration 10 .

Fig. 19 Configurational space for node 1 at iterations 1,5 , and 10 


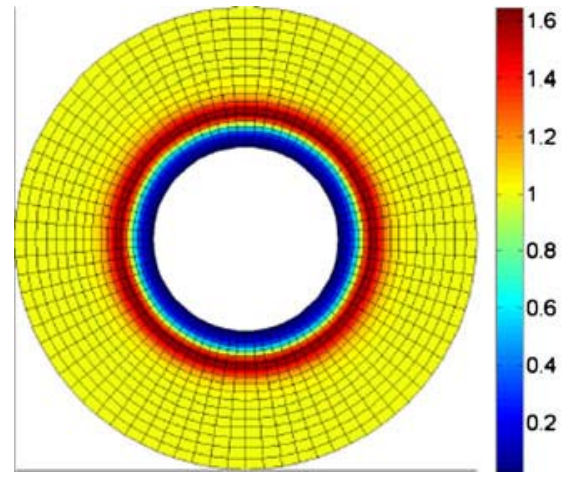

(a) Iteration 1 .

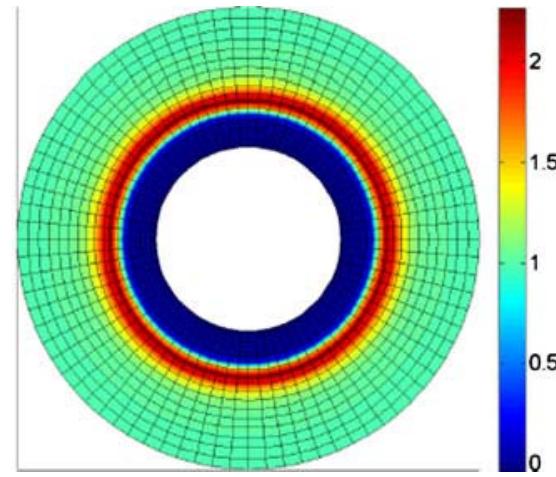

(b) Iteration 5 .

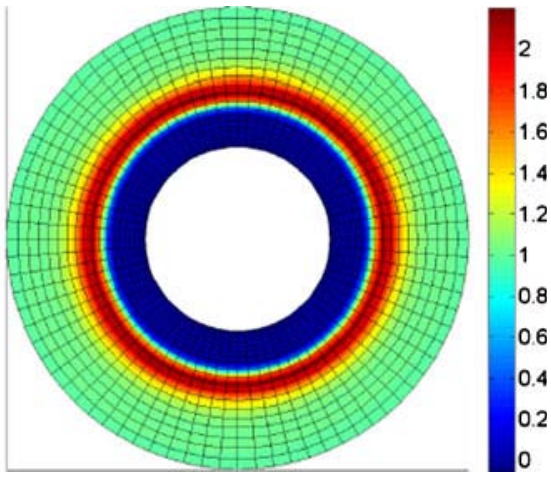

(c) Iteration 10 .

Fig. 20 Configurational space for node 2 at iterations 1, 5, and 10

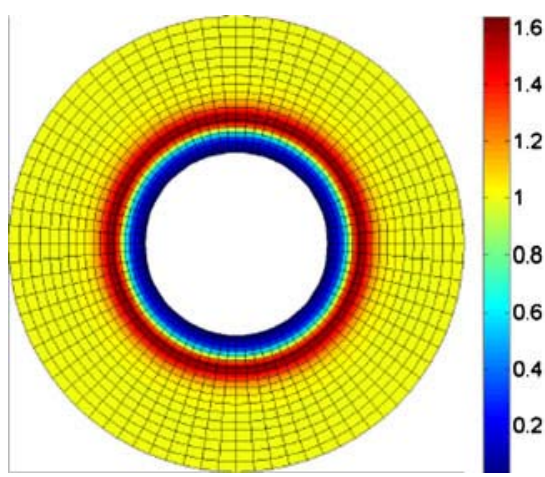

(a) Iteration 1 .

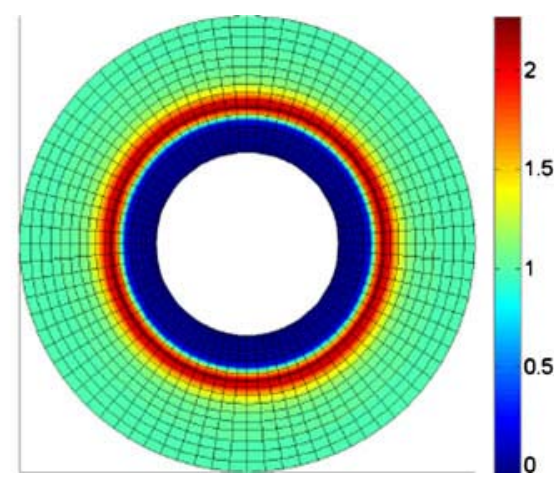

(b) Iteration 5 .

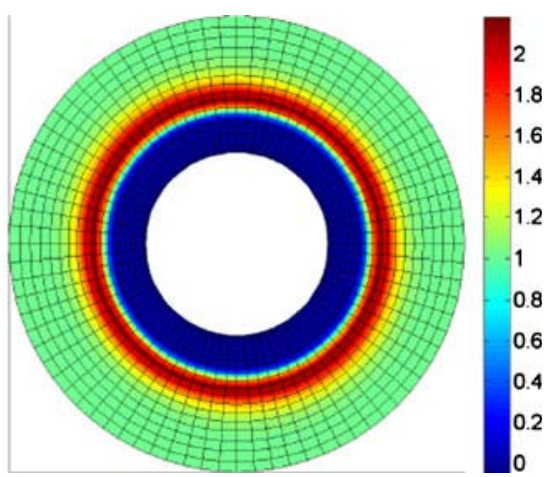

(c) Iteration 10 .

Fig. 21 Configurational space for node 3 at iterations 1, 5, and 10

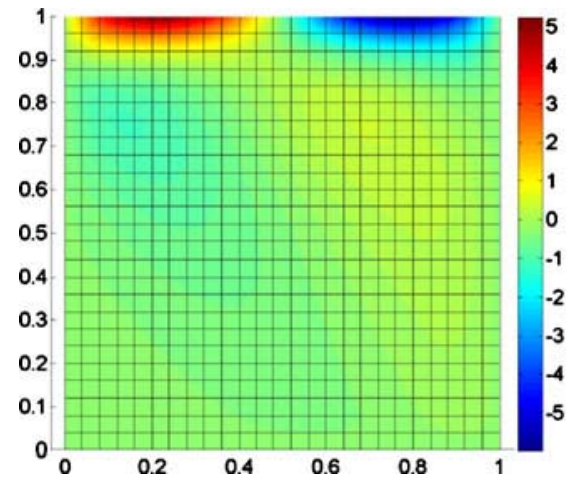

(a) Iteration 1 .
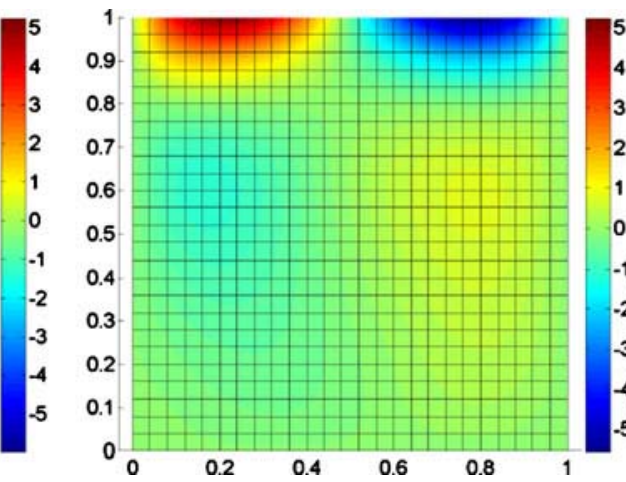

(b) Iteration 5 .

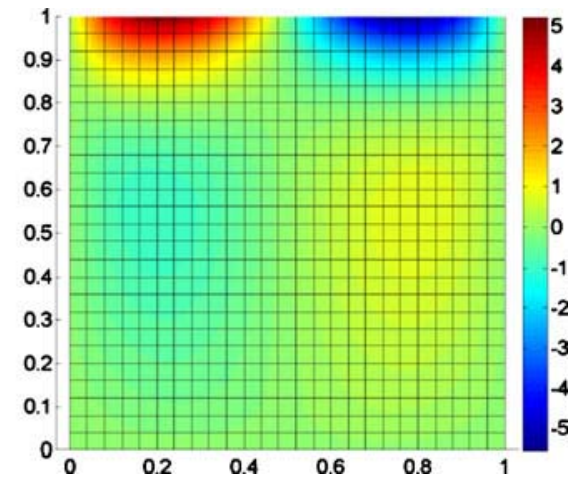

(c) Iteration 10 .

Fig. $22\left(\sigma_{22}-\sigma_{11}\right)$ at iteration 1,5 , and 10 


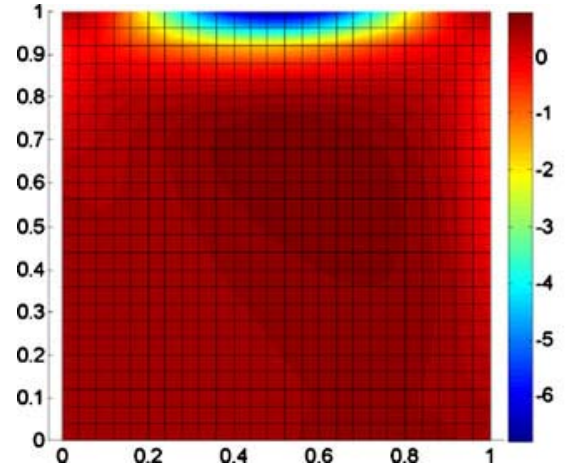

(a) Iteration 1

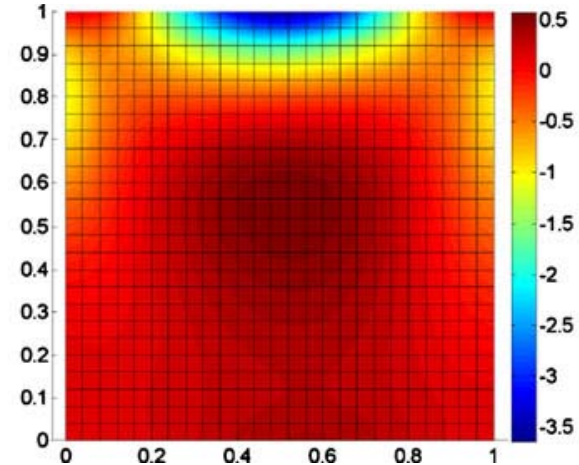

(b) Iteration 5 .

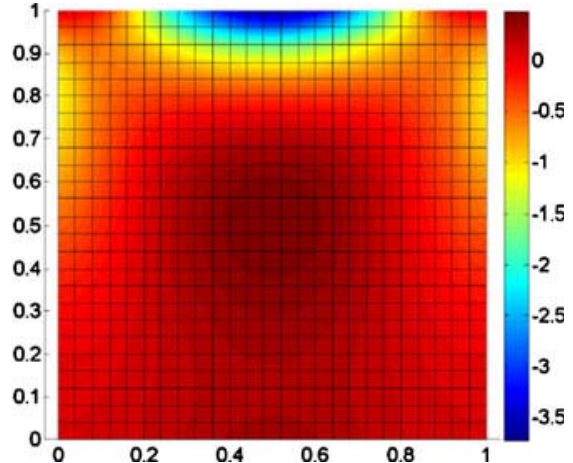

(c) Iteration 10

Fig. $23 \sigma_{12}$ at iteration 1,5 , and 10

As we can notice in Figs. 19, 20, and 21, the more is the velocity gradient, the higher is the anisotropy of the microstructure. For the node 1 that is located in a zone exhibiting large shear rates, flow-induced anisotropy is noticed from the first time step, whereas the distribution in the other nodes remains essentially isotropic.

Figures 22 and 23 depict the effects of the suspension on the stress at iteration 1,5 , and 10 .

\section{Conclusion}

The most direct approach to investigations of colloidal suspensions is the approach adopted in stochastic simulations. Smoluchowski's mesoscopic theory provides an alternative setting. The microstructure in the Smoluchowski theory is characterized by the pair correlation function. In this paper, the PGD was used for solving the Smoluchowski equation. Because the PGD numerical complexity scales linearly with the dimension of the space instead of the exponential growing characteristic of mesh-based discretizations, PGD allows circumventing the curse of dimensionality that models defined in high-dimensional spaces (like the ones related to the Smoluchowski equation) exhibit. Thus, by applying the PGD, we can avoid the solution of the equation governing the evolution of the second-order moment related to the pair correlation function and then the issues related to the closure approximation that such an approach involves.

In this paper, we proved the capabilities of simulating complex flows within the kinetic theory framework. However, we are conscious that the model here considered is not the more pertinent for addressing scenarios of real interest because such scenarios imply taking into account particle migration as well as other ignored effects essentially in concentrated regimes. These topic will be addressed in future works.

\section{Appendix 1: Kinetic coefficients}

The kinetic coefficients $\boldsymbol{\Lambda}$ and $\lambda$ derive from the Stokes problem (motion of rigid spheres in a Newtonian fluid) and appear in Eq. 4. They are defined in Zmievski et al. (2005) by:

$\Lambda_{\alpha \beta}=\frac{k_{\mathrm{B}} T}{3 \pi \eta_{0} a}\left[\sigma_{1}(R / a) \frac{R_{\alpha} R_{\beta}}{R^{2}}+\sigma_{2}(R / a)\left(\delta_{\alpha \beta}-\frac{R_{\alpha} R_{\beta}}{R^{2}}\right)\right]$

$\lambda_{\alpha \beta \gamma}=\left[\mu_{1}(R / a) \frac{R_{\alpha} R_{\beta}}{R^{2}}+\mu_{2}(R / a)\left(\delta_{\alpha \beta}-\frac{R_{\alpha} R_{\beta}}{R^{2}}\right)\right] R_{\gamma}$

The dimensionless form of the above terms is given by

$\Lambda_{\alpha \beta} \rightarrow \frac{3 \pi \eta_{0} a}{k_{\mathrm{B}} T} \Lambda_{\alpha \beta}$ and $\lambda_{\alpha \beta \gamma} \rightarrow \frac{1}{a} \lambda_{\alpha \beta \gamma}$

In this paper, we strictly follow the approximations of the coefficient proposed by Zmievski et al. in Zmievski et al. (2005) (based on Batchelor (1977) and Batchelor and Green (1972)):

$\sigma_{1} \approx 1-\frac{3}{2} \frac{a}{R}+\frac{a^{3}}{R^{3}}-\frac{15}{4} \frac{a^{4}}{R^{4}}+109.0 \frac{a^{6}}{R^{6}}-236.0 \frac{a^{7}}{R^{7}}$

$\sigma_{2} \approx 1-\frac{3}{4} \frac{a}{R}-\frac{1}{2} \frac{a^{3}}{R^{3}}-10.336 \frac{a^{6}}{R^{6}}$ 
$\mu_{1} \approx 5 \frac{a^{3}}{R^{3}}-8 \frac{a^{5}}{R^{5}}+25 \frac{a^{6}}{R^{6}}-423.712 \frac{a^{7}}{R^{7}}+907.424 \frac{a^{8}}{R^{8}}$

$\mu_{2} \approx-\frac{16}{3} \frac{a^{5}}{R^{5}}-15.317 \frac{a^{6}}{R^{6}}$

\section{Appendix 2: PGD algorithm}

This appendix describes the general PGD framework for solving a model defined in a space of dimension $N$ whose discrete form reads:

$\mathcal{A G}=\mathcal{B}$

where

$\mathcal{A}=\sum_{j=1}^{n_{A}} \mathbb{A}_{1}^{j} \otimes \mathbb{A}_{2}^{j} \otimes \ldots \otimes \mathbb{A}_{N}^{j}$

$\mathcal{B}=\sum_{j=1}^{n_{B}} \mathbb{B}_{1}^{j} \otimes \mathbb{B}_{2}^{j} \otimes \ldots \otimes \mathbb{B}_{N}^{j}$

We are looking for the solution as a tensor product of functions

$\mathcal{G}=\sum_{j=1}^{\infty} \mathbb{G}_{1}^{j} \otimes \mathbb{G}_{2}^{j} \otimes \ldots \otimes \mathbb{G}_{N}^{j}$

For computing the involved functions, we are minimizing the residual

$\mathcal{J}(\mathcal{G})=\|\mathcal{A} \mathcal{G}-\mathcal{B}\|_{2}$

that could be rewritten

$$
\begin{aligned}
\mathcal{J}(\mathcal{G}) & =\left(\mathcal{G}-\mathcal{A}^{-1} \mathcal{B}\right)^{T} \mathcal{A}^{T} \mathcal{A}\left(\mathcal{G}-\mathcal{A}^{-1} \mathcal{B}\right) \\
& =\left\|\mathcal{G}-\mathcal{A}^{-1} \mathcal{B}\right\|_{\mathcal{A}^{T} \mathcal{A}}
\end{aligned}
$$

Thus, by differentiation in relation to the field $\mathcal{G}$, the new problem to be solved writes

$\partial \mathcal{G} \mathcal{A}^{T} \mathcal{A} \mathcal{G}=\partial \mathcal{G} \mathcal{A}^{T} \mathcal{B}$

The adaptive strategy consists of enriching progressively the separated representation. Thus, at iteration $n_{G}+1$, we look for:

$\mathcal{G}=\underbrace{\sum_{j=1}^{n_{G}} \mathbb{G}_{1}^{j} \otimes \mathbb{G}_{2}^{j} \otimes \ldots \otimes \mathbb{G}_{N}^{j}}_{\mathcal{G}_{F}}+\underbrace{\mathbb{R}_{1} \otimes \mathbb{R}_{2} \otimes \ldots \otimes \mathbb{R}_{N}}_{\mathcal{G}_{R}}$

Because of the non-linearity, these functions are computed by using an alternating directions fixed point algorithm that proceeds by calculating $\mathbb{R}_{j}$ assuming all the others are known. Thus, when we are looking for $\mathbb{R}_{j}$, the following test function is used

$\mathcal{G}^{*}=\mathbb{R}_{1} \otimes \ldots \otimes \mathbb{R}_{j-1} \otimes \mathbb{R}_{j}^{*} \otimes \mathbb{R}_{j+1} \otimes \ldots \otimes \mathbb{R}_{N}$

The system to be solved then writes

$\mathcal{G}^{*} \mathcal{A}^{T} \mathcal{A} \mathcal{G}_{R}+\mathcal{G}^{*} \mathcal{A}^{T} \mathcal{A G}_{F}=\mathcal{G}^{*} \mathcal{A}^{T} \mathcal{B}$

that results in

$$
\begin{aligned}
& \sum_{i=1}^{n_{F}} \sum_{k^{\prime}=1}^{n_{A}} \sum_{k=1}^{n_{A}}\left(\begin{array}{c}
\mathbb{A}_{j}^{k^{\prime}} \mathbb{A}_{j}^{k} \mathbb{G}_{j}^{i} \prod_{\substack{h=1 \\
h \neq j}}^{N} \mathbb{R}_{h}^{T} \mathbb{A}_{h}^{k^{\prime}} \mathbb{A}_{h}^{k} \mathbb{G}_{h}^{i} \\
\left(\begin{array}{c} 
\\
h
\end{array}\right.
\end{array}\right)
\end{aligned}
$$

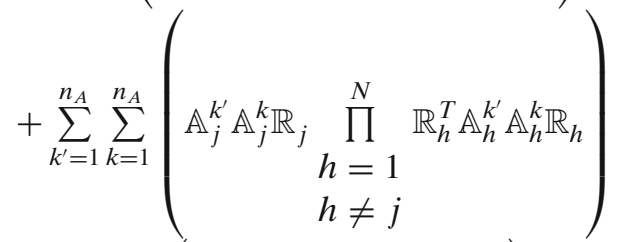

$$
\begin{aligned}
& =\sum_{k^{\prime}=1}^{n_{A}} \sum_{k=1}^{n_{B}}\left(\begin{array}{c}
\mathbb{A}_{j}^{k^{\prime}} \mathbb{B}_{j}^{k} \prod_{\substack{h=1 \\
h \neq j}}^{N} \mathbb{R}_{h}^{T} \mathbb{A}_{h}^{k^{\prime}} \mathbb{B}_{h}^{k} \\
h \neq j
\end{array}\right)
\end{aligned}
$$

\section{Appendix 3: Writing coefficient under separate form}

Some of the coefficients of the pair correlation function are defined both on physical and conformational space, and following the PGD algorithm (see "Appendix 2"), it is needed to rewrite these coefficients in a separated form. Let $A(\boldsymbol{x}, \boldsymbol{R})$ be such a coefficient :

$$
A(\boldsymbol{x}, \boldsymbol{R})=\sum_{i} B_{i}(\boldsymbol{x}) C_{i}(\boldsymbol{R})
$$

However, it is not easy to write the pair correlation function coefficients in a separate form. As the only dependence of these coefficients on the physical coordinates is through the velocity gradient, we could express it in the velocity gradient canonical basis. In that basis, the velocity gradient writes (in 2D for the sake of simplicity):

$$
\begin{aligned}
\frac{\partial \boldsymbol{v}}{\partial \boldsymbol{x}}= & \left(\begin{array}{ll}
\frac{\partial v_{1}}{\partial x_{1}} \frac{\partial v_{1}}{\partial x_{2}} \\
\frac{\partial v_{2}}{\partial x_{1}} \frac{\partial v_{2}}{\partial x_{2}}
\end{array}\right)=\frac{\partial v_{1}}{\partial x_{1}}\left(\begin{array}{ll}
1 & 0 \\
0 & 0
\end{array}\right)+\frac{\partial v_{1}}{\partial x_{2}}\left(\begin{array}{ll}
0 & 1 \\
0 & 0
\end{array}\right) \\
& +\frac{\partial v_{2}}{\partial x_{1}}\left(\begin{array}{ll}
0 & 0 \\
1 & 0
\end{array}\right)+\frac{\partial v_{2}}{\partial x_{2}}\left(\begin{array}{ll}
0 & 0 \\
0 & 1
\end{array}\right)
\end{aligned}
$$


$A(\boldsymbol{x}, \boldsymbol{R})$ can be rewritten as:

$$
\begin{aligned}
A(\boldsymbol{x}, \boldsymbol{R})= & \frac{\partial v_{1}}{\partial x_{1}}(\boldsymbol{x}) A^{11}(\boldsymbol{R})+\frac{\partial v_{1}}{\partial x_{2}}(\boldsymbol{x}) A^{12}(\boldsymbol{R}) \\
& +\frac{\partial v_{2}}{\partial x_{1}}(\boldsymbol{x}) A^{21}(\boldsymbol{R})+\frac{\partial v_{2}}{\partial x_{2}}(\boldsymbol{x}) A^{22}(\boldsymbol{R}) \\
= & \sum_{i=1}^{2} \sum_{j=1}^{2} \frac{\partial v_{i}}{\partial x_{j}}(\boldsymbol{x}) A^{i j}(\boldsymbol{R})
\end{aligned}
$$

where all the terms $A^{i j}(\boldsymbol{R})$ are determined numerically.

\section{References}

Ammar A, Mokdad B, Chinesta F, Keunings R (2006) A new family of solvers for some classes of multidimensional partial differential equations encountered in kinetic theory modeling of complex fluids. J Non-Newton Fluid Mech 139(3):153

Ammar A, Mokdad B, Chinesta F, Keunings R (2007) A new family of solvers for some classes of multidimensional partial differential equations encountered in kinetic theory modelling of complex fluids: Part II: Transient simulation using space-time separated representations. J Non-Newton Fluid Mech 144(2-3):98

Ammar A, Pruliere E, Chinesta F, Laso M (2009) Reduced numerical modeling of flows involving liquid-crystalline polymers. J Non-Newton Fluid Mech 160(2-3):140

Batchelor G (1977) Effect of Brownian-motion on bulk stress in a suspension of spherical-particles. J Fluid Mech 83(Nov):97

Batchelor GK, Green JT (1972) The hydrodynamic interaction of two small freely-moving spheres in a linear flow field. J Fluid Mech 56:375

Bergenholtz J, Brady J, Vicic M (2002) The non-Newtonian rheology of dilute colloidal suspensions. J Fluid Mech 456(1):239

Brady JF (2001) Computer simulation of viscous suspensions. Chem Eng Sci 56(9):2921

Brady J, Morris J (1997) Microstructure of strongly sheared suspensions and its impact on rheology and diffusion. J Fluid Mech 348:103

Brady JF, Bossis G (1988) Stokesian dynamics. Annu Rev Fluid Mech 20:111
Foss D, Brady J (2000) Structure, diffusion and rheology of Brownian suspensions by Stokesian dynamics simulation. J Fluid Mech 407:167

Gao C, Kulkarni S, Morris J, Gilchrist J (2010) Direct investigation of anisotropic suspension structure in pressure-driven flow. Phys Rev E 81(4):041403

Kolli V, Pollauf E, Gadala-Maria F (2002) Transient normal stress response in a concentrated suspension of spherical particles. J Rheol 46(1):321

Lionberger R, Russel W (1997) A Smoluchowski theory with simple approximations for hydrodynamic interactions in concentrated dispersions. J Rheol 41:399

Miller R, Singh J, Morris J (2009) Suspension flow modeling for general geometries. Chem Eng Sci 64(22):4597

Morris J (2009) A review of microstructure in concentrated suspensions and its implications for rheology and bulk flow. Rheol Acta 48(8):909

Morris J, Katyal B (2002) Microstructure from simulated Brownian suspension flows at large shear rate. Phys Fluids 14:1920

Nouy A (2010) A priori model reduction through Proper Generalized Decomposition for solving time-dependent partial differential equations. Comput Methods Appl Mech Eng 199(23-24):1603

Ohl N, Gleissle W (1993) The characterization of the steady-state shear and normal stress functions of highly concentrated suspensions formulated with viscoelastic liquids. J Rheol 37(2):381

Phan-Thien N (1995) Constitutive equation for concentrated suspensions in Newtonian liquids. J Rheol 39(4):679

Phung T, Brady J, Bossis G (1996) Stokesian dynamics simulation of Brownian suspensions. J Fluid Mech 313:181

Smoluchowski MV (1915) Über Brownsche Molekularbewegung unter Einwirkung äußerer Kräfte und den Zusammenhang mit der verallgemeinerten Diffusionsgleichung. Ann Phys 353:1103

So J, Yang S, Hyun J (2001) Microstructure evolution and theological responses of hard sphere suspensions. Chem Eng Sci 56(9):2967

Stickel J, Powell R (2005) Fluid mechanics and rheology of dense suspensions. Annu Rev Fluid Mech 37:129

Stickel J, Phillips R, Powell R (2006) A constitutive model for microstructure and total stress in particulate suspensions. $\mathrm{J}$ Rheol 50:379

Wilson H (2005) An analytic form for the pair distribution function and rheology of a dilute suspension of rough spheres in plane strain flow. J Fluid Mech 534(1):97

Zmievski V, Grmela M, Bousmina M, Dagréou S (2005) Nonlinear microstructure and rheology of semidilute colloidal suspensions of structureless particles. Phys Rev E Stat Nonlinear Soft Matter Phys 71(5 Pt 1):051503 